\title{
Subjective expected utility in games
}

\author{
Alfredo Di Tillio \\ Department of Economics and IGIER, Università Bocconi
}

\begin{abstract}
This paper extends Savage's subjective approach to probability and utility from decision problems under exogenous uncertainty to choice in strategic environments. Interactive uncertainty is modeled both explicitly, using hierarchies of preference relations, the analogue of beliefs hierarchies, and implicitly, using preference structures, the analogue of type spaces à la Harsanyi, and it is shown that the two approaches are equivalent. Preference structures can be seen as those sets of hierarchies arising when certain restrictions on preferences, along with the players' common certainty of the restrictions, are imposed. Preferences are a priori assumed to satisfy only very mild properties (reflexivity, transitivity, and monotone continuity). Thus, the results provide a framework for the analysis of behavior in games under essentially any axiomatic structure. An explicit characterization is given for Savage's axioms, and it is shown that a hierarchy of relatively simple preference relations uniquely identifies the decision maker's utilities and beliefs of all orders. Connections with the literature on beliefs hierarchies and correlated equilibria are discussed.

KeYwords. Subjective probability, preference hierarchies, type spaces, beliefs hierarchies, common belief, expected utility, incomplete information, correlated equilibria.
\end{abstract}

JEL CLASSIFICATION. C70, D80, D81, D82, D83.

\section{INTRODUCTION}

This paper concerns choice in strategic environments, where the hypothesis that an agent is a subjective expected utility (henceforth SEU) maximizer, believes each other agent is, believes each other agent believes each other agent is, and so on, is not assumed, but rather must be derived, as in Savage (1954), from rules on subjective preference.

A game situation specifies a set $Z$ of outcomes and a set $S$ describing the possible states of nature and strategies of the players. Starting from these primitives, ${ }^{1}$ one

Alfredo Di Tillio: alfredo.ditillio@unibocconi.it

This paper is a very substantial revision of the third chapter of my Ph.D. thesis at the University of Pennsylvania. I am grateful to George Mailath for his invaluable input and advice. I would also like to thank Pierpaolo Battigalli and Larry Epstein for many helpful conversations, as well as Jeffrey Ely and three anonymous referees for their detailed and very useful comments.

${ }^{1}$ Of course, in order to obtain a game form one must specify an outcome function $\zeta: S \rightarrow Z$, but we do not need this; as in Savage's analysis, we assume that all acts-mappings from $S$ to $Z$-are in principle available. Also, we include a player's strategy and preferences in that player's own uncertainty; as we explain later in the paper, our results remain true without this assumption.

Copyright (c) 2008 Alfredo Di Tillio. Licensed under the Creative Commons Attribution-NonCommercial License 3.0. Available at http: / / econtheory . org. 
analyzes the players' behavior and interactive beliefs using a type space à la Harsanyi (1967-68), i.e. an abstract space $X$ and, for every point in the space, a state of nature as well as a strategy, a utility function on $Z$, and a probability measure on $X$ for each player. Indeed, each point of $X$ generates a beliefs hierarchy specifying each player's first-order belief, i.e. belief over $S$, second-order belief, i.e. belief over $S$ and all players' first-order beliefs, and so on. Mertens and Zamir (1985) prove that (i) every beliefs hierarchy is generated by some type space and (ii) a set of hierarchies is generated by some type space if and only if it arises from the space of all hierarchies when we impose the players' common belief (concerning some event in the space of all hierarchies itself).

How can such a model be justified in terms of preferences? Note that once we specify a utility function for a player, then this player is able to rank "acts" whose outcome depends on the players' $n$-order beliefs according to expected utility, via $(n+1)$-order beliefs. In a seminal work, Epstein and Wang (1996) propose to generalize this by allowing more general preference hierarchies, specifying a player's first-order preferences, i.e. preferences over acts that depend on $S$, second-order preferences, i.e. preferences over acts that depend on $S$ and first-order preferences, and so on. Beliefs hierarchies and other models can then be accommodated within this framework by considering appropriate subsets of hierarchies, obtained after imposing the players' common certainty (generalized common belief using the notion of Savage-null event).

In Epstein and Wang's paper, $Z$ is assumed to be the interval $[0,1]$, the space $S$ is assumed to be compact Hausdorff, and preferences are a priori restricted to be complete, transitive, monotonic, and regular-see Epstein and Wang (1996) for details. This paper provides an alternative yet analogous construction where $S$ and $Z$ are assumed to be finite but preferences are considerably less restricted—in particular, they are a priori assumed to satisfy only reflexivity and transitivity.2 Our main contribution is to show that, while indeed allowing the more general, almost axiomless construction and theorems, the said finiteness assumption still delivers a framework rich enough to accommodate a large class of type spaces, namely, all those whose underlying space $X$ is Polish and for which the beliefs are non-atomic. (Such type spaces arise naturally even with a finite space of basic uncertainty and even with trivial second- and higher-order beliefs, as long as a continuum of first-order beliefs is allowed-see Section 6.9.) This is somewhat surprising, because such type spaces are suitable to generate all possible finite order beliefs, and while our finite order preferences are always about finite sets of possible acts, each mapping a finite state space into $Z$, they are still able to distinguish different finite order beliefs, no matter how detailed these beliefs are or how high their order is-see Section 6.9 for an illustration.

\footnotetext{
${ }^{2}$ The space of hierarchies we construct is such that every preference relation on acts defined on this space automatically satisfies a third axiom, monotone continuity (see Villegas 1964 and Arrow 1970). This proves useful in Sections 4 and 5, where we discuss common certainty. Indeed, this requires preferences to uniquely extend to a larger family of acts, and while there are many such extensions, we prove that a unique monotone continuous extension exists. In this sense, the results in those sections depend on this third axiom as well. Monotone continuity is equivalent to countable additivity of the belief in the Savage representation if this exists; we prove (Lemma 7 in Appendix C) that it guarantees the analogous property even in the absence of axioms other than transitivity.
} 


\section{Outline of the analysis and plan of the paper}

Let $I$ be the finite set of players. The key notion introduced in this paper is that of preference structure, defined by an abstract set $X$, a function $\sigma: X \rightarrow S$, and a function $\vartheta^{i}: X \rightarrow \Pi(X)$ for each $i \in I$, where $\Pi(X)$ is the set of preference relations on the family of maps from $X$ to $Z{ }^{3}$ This model implicitly defines a set of preference hierarchies, just as a type space à la Harsanyi implicitly defines a set of beliefs hierarchies. ${ }^{4}$ Indeed, each point in $X$ implicitly describes every player's preference over acts of the form $f: S \rightarrow Z$, acts of the form $f: S \times \Pi^{I}(S) \rightarrow Z$, acts of the form $f: S \times \Pi^{I}(S) \times \Pi^{I}\left(S \times \Pi^{I}(S)\right) \rightarrow Z$, and so on. ${ }^{5}$ Thus a preference structure, like a type space, is an abstract object encoding a set of hierarchies. As such, the model raises questions analogous to those arising for type spaces, and this paper is devoted to answering some basic ones.

The most basic questions concern the generality of the model. On one hand, full generality is desirable: Are all possible hierarchies generated by some structure, and more generally, what characterizes the sets of hierarchies that can be described by means of structures? On the other hand, just full generality is sometimes desirable: In what cases, and in what sense, are two structures describing the same hierarchies equivalent? In Section 3 we show, analogously to Mertens and Zamir's (1985) main theorem, that our model carries no loss of generality. The set $\Omega$ of all sequences comprising an element of $S$ and a preference hierarchy for each player is such that every $\omega \in \Omega$ corresponds to a unique preference relation $\varpi^{i}(\omega)$ for each $i \in I$ on the set of acts of the form $f: \Omega \rightarrow Z$. Indeed, $\Omega$ is isomorphic (in a natural sense) to $S \times \Pi^{I}(\Omega)$. Furthermore, the canonical preference structure comprising the space $\Omega$, the natural projection of $\Omega$ on $S$, and the mappings $\varpi^{i}$, is such that every other preference structure can be mapped into it in a unique way. Finally, any set of hierarchies that can be generated by some structure is, in fact, isomorphic to every structure that generates that set and satisfies minimality and non-redundancy; in particular, any two minimal and non-redundant structures generating the same hierarchies are isomorphic.

The other main questions concern the interpretation and applicability of the model, with special emphasis on SEU. Can we interpret a preference structure (e.g. one where only SEU preferences appear) as a situation where some event (i.e. some subset of $\Omega$, e.g. the fact that all players have SEU preferences) obtains and this is common certainty among the players? Conversely, given an arbitrary event, can we always find a preference structure that is isomorphic to the set of hierarchies where that event obtains and this

\footnotetext{
${ }^{3}$ Epstein and Wang (1996) adopt an approach analogous to that of Brandenburger and Dekel (1993), focusing on the extension results and the consequent isomorphism results. However, it does not address the question of whether, how exactly, and why in modeling a concrete strategic scenario one can without loss of generality assume a Harsanyi type space-like construct-though objects similar to our preference structures are indeed briefly discussed as an illustration (see page 1348 of Epstein and Wang 1996). In Section 6.1 we sketch how this question can be answered in Epstein and Wang's framework.

${ }^{4}$ More precisely, the analogy is with a model à la Aumann (1987), where strategies appear explicitly in the description of a state. Indeed, our notion of preference structure logically corresponds to what Aumann calls "information system" in Aumann (1987). See also Section 6.8.

${ }^{5}$ In this paper we do not work with the full set $S \times \Pi^{I}(S) \times \Pi^{I}\left(S \times \Pi^{I}(S)\right) \times \cdots$. Instead, we impose coherency at all levels of the hierarchies. However, the alternative construction where hierarchies are unrestricted and coherency is imposed a posteriori is entirely equivalent—see Di Tillio (2006) for a proof.
} 
is common certainty? This identification is not always granted, but we show that under non-redundancy it does hold in two (mutually nonexclusive) cases. The first case, analyzed in Section 4, is when we assume a simple structure-this means that its events form a countable base for a compact Hausdorff topology. The main result in that section states that a non-redundant structure is simple if and only if isomorphic to a closed component, i.e. a set of hierarchies obtained after imposing an intersection of basic events in $\Omega$ along with common certainty of it. (Examples of simple spaces are given in Section 6.3.) The second case, analyzed in Section 5, is when we assume a standard Savage structure-this means that its underlying space is standard Borel and all preferences satisfy Savage's SEU axioms. The main result in that section states that a non-redundant structure is standard Savage if and only if isomorphic to a Savage component, namely, a set of hierarchies obtained after imposing Savage's SEU axioms, along with some event in the $\sigma$-algebra generated by the basic events in $\Omega$, and common belief (Savage's axioms plus common certainty) of this. ${ }^{6}$ In Section 5 we also prove that standard Savage structures are completely equivalent objects to standard Savage type spaces, i.e. type spaces à la Harsanyi where the underlying space is standard Borel and all beliefs are non-atomic.

Section 6 contains some clarifications and discussions and provides some noteworthy extensions to our results. All proofs are relegated to the various appendices.

\section{Preliminaries}

The primitives of our model are a finite set $Z$ of outcomes, a finite set $S^{0}$ of states of nature, a finite set $I$ of players, and for each player $i$ a finite set $S^{i}$ of strategies. We define $S=\times_{j \in\{0\} \cup I} S^{j}$ and, to avoid trivialities, we assume $|Z| \geq 2 .{ }^{7}$

An uncertainty space or simply a space is a pair $(X, \mathscr{B})$ where $X$ is a set and $\mathscr{B}$ is an algebra of subsets of $X$ called events. If the specific $\mathscr{B}$ is irrelevant or clear from the context, we may refer to $X$ alone as a space; for instance, if $X$ is finite, it is understood to be endowed with the algebra of all its subsets. Any subset $X^{\prime}$ of $X$ equipped with its relative algebra, i.e. the family of all sets of the form $X^{\prime} \cap E$ where $E \in \mathscr{B}$, is a subspace of $X$.

An act is a function $f: X \rightarrow Z$ such that $f^{-1}(z) \in \mathscr{B}$ for all $z \in Z$. The act mapping every $x \in X$ to the same $z \in Z$ is denoted $z$. Given two acts $f, g$ and an event $E$, we write $f E g$ for the act that coincides with $f$ on $E$ and with $g$ on $X \backslash E$. The set of all

\footnotetext{
${ }^{6}$ The class of events in $\Omega$ that makes the universality results of Section 3 hold is the algebra $\mathscr{A}$ generated by those sets of hierarchies whose elements share the same $n$-order preferences for some $n$. (In other words, it is the algebra of cylinders on $\Omega$.) These basic events are, in themselves, too coarse to meaningfully discuss common certainty, so for the latter one needs to extend preferences (as already mentioned in footnote 2) to acts measurable with respect to a larger family of acts. The extension to arbitrary intersections of events in $\mathscr{A}$ is always possible by monotone continuity (Proposition 5), whereas the extension to events in the $\sigma$-algebra generated by $\mathscr{A}$, which is needed in order to discuss common certainty of Savage's axioms, is possible under Savage's axioms themselves (Proposition 7). Further discussion and explanations are provided in Section 3.5.

${ }^{7}$ All results in the paper hold as long as there are at least two outcomes. States of nature and strategies can be singletons. This is because whether a player prefers one outcome to another already creates some non-trivial first level uncertainty on which to build.
} 
acts is denoted $F(X, \mathscr{B})$ or just $F(X)$ if the specific $\mathscr{B}$ needs no emphasis. Note that $F(X)$ is countable if $\mathscr{B}$ is countable. ${ }^{8}$ If $\pi$ is a binary relation on $F(X)$, i.e. a subset of $F(X) \times F(X)$, then for all $f, g \in F(X)$ we write $(f, g) \dot{\epsilon} \pi$ as an abbreviation for $(f, g) \in \pi \not \supset$ $(g, f)$. An event $E$ is null according to $\pi$, or just $\pi$-null, if $(f, g E f) \in \pi$ for all $f, g \in F(X)$. A preference relation on $F(X)$ is a reflexive and transitive binary relation on $F(X)$. The set of all preference relations on $F(X, \mathscr{B})$ is denoted $\Pi(X, \mathscr{B})$ and is always assumed to be endowed with the algebra generated by the sets of the form

$$
\{\pi \in \Pi(X, \mathscr{B}):(f, g) \in \pi\}
$$

where $f, g \in F(X, \mathscr{B})$. (See Section 6.2 for a discussion.) The space $\times_{i \in I} \Pi(X, \mathscr{B})$ is abbreviated $\Pi^{I}(X, \mathscr{B})$. If reference to the specific algebra $\mathscr{B}$ is superfluous, we may write $\Pi(X)$ instead of $\Pi(X, \mathscr{B})$ and $\Pi^{I}(X)$ instead of $\Pi^{I}(X, \mathscr{B})$. Note that $\Pi(X)$ is finite whenever $X$ is finite, and in this case the algebra on $\Pi(X)$ specified above is precisely the algebra of all subsets of $\Pi(X)$. Given another space $(Y, \mathscr{C})$, a map $\varphi: X \rightarrow Y$ is measurable $\mathscr{B} / \mathscr{C}$ (or just measurable) if $\varphi^{-1}(E) \in \mathscr{B}$ for all $E \in \mathscr{C}$. This induces a measurable function $\widehat{\varphi}: \Pi(X) \rightarrow \Pi(Y)$, namely,

$$
\pi \mapsto\{(f, g) \in F(Y) \times F(Y):(f \circ \varphi, g \circ \varphi) \in \pi\}
$$

For any (not necessarily measurable) map $\phi: Y \rightarrow \Pi^{I}(X)$ and any player $i$, we write $\phi^{i}$ for the function from $Y$ to $\Pi(X)$ that maps $y \in Y$ to the $i$ th coordinate of $\phi(y)$.

\section{PREFERENCE HIERARCHIES AND PREFERENCE STRUCTURES}

In this section we construct the space of all coherent preference hierarchies and prove its basic properties. We also introduce preference structures, a convenient way of describing sets of such hierarchies implicitly. In our construction, a player's strategy and preferences appear in that player's own uncertainty; a player is not assumed to know (in either a formal or informal sense) her own strategy or preferences. While this gives also a more general construction, we do it mainly for notational convenience, i.e. to avoid having to construct different spaces of hierarchies for different players. (We explain this further in Section 6.7 below.)

\subsection{The space of coherent preference hierarchies}

Define $\Omega_{0}=S$ and $\Omega_{1}=\Omega_{0} \times \Pi^{I}\left(\Omega_{0}\right)$. Let $\rho_{0}: \Omega_{1} \rightarrow \Omega_{0}$ and $\delta_{1}: \Omega_{1} \rightarrow \Pi^{I}\left(\Omega_{0}\right)$ be the natural projections, and let $\widehat{\rho}_{0}: \Pi\left(\Omega_{1}\right) \rightarrow \Pi\left(\Omega_{0}\right)$ be the mapping induced by $\rho_{0}$. Proceeding recursively, define $\Omega_{n+1}$ for $n \geq 1$ as the largest subspace of $\Omega_{n} \times \Pi^{I}\left(\Omega_{n}\right)$ such that the following diagram-where $\rho_{n}: \Omega_{n+1} \rightarrow \Omega_{n}$ and $\delta_{n+1}: \Omega_{n+1} \rightarrow \Pi^{I}\left(\Omega_{n}\right)$ are the natural projections, as before-commutes for every player $i$ :

${ }^{8}$ As $Z$ is finite, the set of constant acts is finite, and every act whose range has at most $n \geq 2$ distinct outcomes can be written as $z E f$, where $z \in Z, E \in \mathscr{B}$, and the range of $f$ has at most $n-1$ outcomes. 


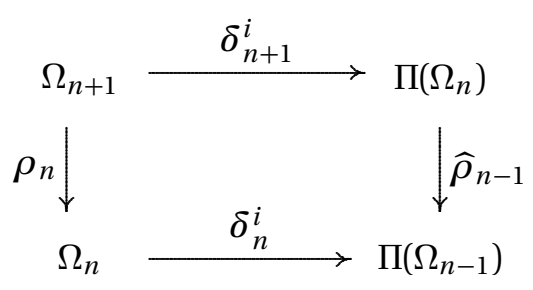

To complete the recursion, let $\widehat{\rho}_{n}$ be the map from $\Pi\left(\Omega_{n+1}\right)$ to $\Pi\left(\Omega_{n}\right)$ induced by $\rho_{n} \cdot{ }^{9}$

The space of coherent preference hierarchies (or simply hierarchies) is the set

$$
\Omega=\left\{\left(\omega_{0}, \omega_{1}, \ldots\right) \in \Omega_{0} \times \Omega_{1} \times \cdots: \rho_{n}\left(\omega_{n+1}\right)=\omega_{n} \forall n \geq 0\right\}
$$

equipped with the algebra $\mathscr{A}$ of all subsets of the form $\varrho_{n}^{-1}(A)$, where $n \geq 0, A \subseteq \Omega_{n}$, and $\varrho_{n}$ is the natural projection of $\Omega$ on $\Omega_{n} .{ }^{10}$ For all $n \geq 0$, let $\widehat{\varrho}_{n}: \Pi(\Omega) \rightarrow \Pi\left(\Omega_{n}\right)$ be the mapping induced by $\varrho_{n}$. Then we have the following result.

Proposition 1. There exists a unique mapping $\varpi: \Omega \rightarrow \Pi^{I}(\Omega)$ such that $\widehat{\varrho}_{n-1} \circ \varpi^{i}=$ $\delta_{n}^{i} \circ \varrho_{n}$ for every player $i$ and every $n \geq 1$. Furthermore, the mapping $\varpi$ is measurable.

Thus, coherent preference hierarchies are enough to describe the players' strategic uncertainty: a sequence of preference relations on the spaces $F\left(\Omega_{n}\right)$ that is coherent in the sense of the diagram above determines a unique preference relation on $F(\Omega)$. The converse, of course, also holds; any preference relation $\pi \in \Pi(\Omega)$ induces the sequence of preference relations $\widehat{\varrho}_{n}(\pi) \in \Pi\left(\Omega_{n}\right)$, which are clearly coherent. This proposition is analogous to Theorem 5.1 in Epstein and Wang (1996) and parallels the well-known results concerning coherent beliefs hierarchies-see Proposition 2 in Brandenburger and Dekel (1993) and Theorem 2.9 in Mertens and Zamir (1985).

\subsection{Preference structures}

Sets of coherent preference hierarchies can be described more compactly using the following straightforward generalization of Harsanyi's notion of a type space.

Definition 1. A preference structure (or simply a structure) is a tuple $\mathscr{X}=(X, \mathscr{B}, \sigma, \vartheta)$ where $(X, \mathscr{B})$ is a space and $\sigma: X \rightarrow S$ and $\vartheta: X \rightarrow \Pi^{I}(X, \mathscr{B})$ are measurable functions.

This generates a set of hierarchies in a natural way. Let $\gamma_{0}=\sigma$ and let $\widehat{\gamma}_{0}$ be the induced mapping from $\Pi(X, \mathscr{B})$ to $\Pi\left(\Omega_{0}\right)$. For all $n \geq 0$, recursively, let $\gamma_{n+1}: X \rightarrow \Omega_{n+1}$ be

$$
x \mapsto\left(\gamma_{n}(x),\left(\widehat{\gamma}_{n}\left(\vartheta^{i}(x)\right)\right)_{i \in I}\right)
$$

and let $\widehat{\gamma}_{n+1}: \Pi(X) \rightarrow \Pi\left(\Omega_{n+1}\right)$ be the induced mapping. Finally, let $\gamma: X \rightarrow \Omega$ be

$$
x \mapsto\left(\gamma_{0}(x), \gamma_{1}(x), \ldots\right) .
$$

\footnotetext{
${ }^{9}$ Commutativity of the diagram is what we refer to as coherency. We follow Mertens and Zamir's (1985) approach to coherency, whereby the property is built-in rather than being imposed a posteriori (as Epstein and Wang 1996 instead do, following Brandenburger and Dekel 1993). We could have followed the latter route, obtaining essentially the same results obtained here; see Di Tillio (2006) for a formal statement.

${ }^{10}$ By Lemma 5 in Appendix B, $\varrho_{n}$ is onto for all $n \geq 0$, hence $\mathscr{A}$ is indeed a well-defined algebra.
} 
Since $\sigma$ and $\vartheta$ are measurable, and since $\gamma_{n}=\rho_{n} \circ \gamma_{n+1}$ for all $n \geq 0$ by construction, the functions $\gamma_{0}, \gamma_{1}, \ldots$ are all well defined and measurable, hence so is $\gamma$. The latter is the generator of the structure $\mathscr{X}$; its range is the set of hierarchies generated by $\mathscr{X}$.

Thus a preference structure, like a type space à la Harsanyi, provides a convenient, parsimonious description of a set of hierarchies. However, preference structures, like type spaces, are abstract constructs. In particular, the use of such models poses questions that are conceptually analogous to those arising in the context of beliefs hierarchies. First, in what sense can two structures generating the same hierarchies be considered equivalent? Second, what characterizes the sets of hierarchies that can be generated by means of preference structures? Third, can we interpret a preference structure as a situation where certain events (e.g. those disjoint from the one generated by the structure) are ruled out, and there is common certainty of this among the players? In the remainder of this section we provide an answer to the first two questions.

\subsection{Morphisms between structures, minimality and non-redundancy}

In order to formulate equivalence between structures, we need an appropriate notion of isomorphism, whereby two structures generate the same hierarchies if, and (under some conditions) only if, they are isomorphic. The following definition provides the necessary starting point.

Definition 2. A morphism from a preference structure $\mathscr{X}=(X, \mathscr{B}, \sigma, \vartheta)$ to a preference structure $\mathscr{X}^{\prime}=\left(X^{\prime}, \mathscr{B}^{\prime}, \sigma^{\prime}, \vartheta^{\prime}\right)$ is a measurable function $\phi: X \rightarrow X^{\prime}$ such that, letting $\widehat{\phi}: \Pi(X) \rightarrow \Pi\left(X^{\prime}\right)$ denote the mapping induced by $\phi$, the diagram

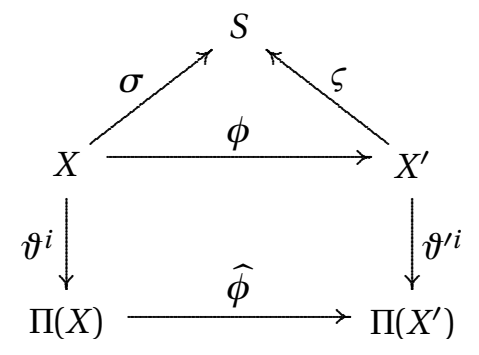

commutes for every player $i$. The structures $\mathscr{X}$ and $\mathscr{X}^{\prime}$ are isomorphic if, in addition, $\phi$ is a bijection and its inverse $\phi^{-1}: X^{\prime} \rightarrow X$ is a morphism from $\mathscr{X}^{\prime}$ to $\mathscr{X}$.

Clearly, isomorphic structures generate the same hierarchies. The converse claim, however, does not hold, because a structure may contain more information than another and yet generate the same hierarchies: a structure can be redundant, i.e. have distinct elements mapped into the same hierarchy by its generator, while the other is not; a structure can be minimal, i.e. have events mapped into events by its generator, while the other is not. ${ }^{11}$ In order to deal with this issue, we first provide formal definitions of

\footnotetext{
${ }^{11}$ Both of these facts have their analogues in the literature on type spaces and raise similar issues there. See Mertens and Zamir (1985) and also the recent papers by Ely and Pęski (2006) and Dekel et al. (2007) on the effect of redundancy on the predictions of rationalizability.
} 
redundancy and minimality that, conveniently, do not explicitly refer to properties of the generator.

Definition 3. Let $\mathscr{X}=(X, \mathscr{B}, \sigma, \vartheta)$ be a structure. Let $\underline{\mathscr{B}}$ be the smallest algebra $\mathscr{B}$ ' such that $^{12}$

$$
\begin{aligned}
\sigma^{-1}(s) \in \mathscr{B}^{\prime} & & \forall s \in S \\
\left\{x \in X:(f, g) \in \vartheta^{i}(x)\right\} \in \mathscr{B}^{\prime} & & \forall i \in I, \forall f, g \in F\left(X, \mathscr{B}^{\prime}\right) .
\end{aligned}
$$

The structure $\mathscr{X}$ is minimal if $\mathscr{B}=\mathscr{\mathscr { B }}$. It is non-redundant if $\mathscr{\mathscr { B }}$ separates the points of $X$. The associated minimal structure is the preference structure $\underline{\mathscr{X}}=(X, \underline{\mathscr{B}}, \sigma, \underline{\vartheta})$ where

$$
\underline{\vartheta}^{i}(x)=\vartheta^{i}(x) \cap[F(X, \underline{\mathscr{B}}) \times F(X, \underline{\mathscr{B}})] \quad \forall i \in I, \forall x \in X .
$$

Analogously to the probabilistic case, non-redundancy requires distinct elements of $X$ to differ in terms of states of nature, strategies, or preferences. Minimality says that those subsets of $X$, which measurability of $\sigma$ and $\vartheta$ requires to belong to $\mathscr{B}$, are the only (basic) events the players can reason about. Indeed, these properties are characterized in terms of the generator as anticipated above.

Proposition 2. A structure $(X, \mathscr{B}, \sigma, \vartheta)$ is minimal if and only if $\mathscr{B}$ is the smallest algebra $\mathscr{B}^{\prime}$ such that the generator of the structure is measurable $\mathscr{B}^{\prime} / \mathscr{A}$. Moreover, whether it is minimal or not, the structure is non-redundant if and only if its generator is injective.

Two minimal and non-redundant structures generate the same set of hierarchies if and only if they are isomorphic. A direct proof of this equivalence is not needed, since necessity (as pointed out earlier) is obvious, whereas sufficiency is an immediate corollary of Proposition 4 below. Thus, we now proceed to answer the second main question left unanswered above, giving a full characterization of the sets of hierarchies that can be generated by means of structures.

\subsection{The canonical preference structure}

Recall that $\varrho_{0}: \Omega \rightarrow S$ and $\varpi: \Omega \rightarrow \Pi^{I}(\Omega)$ denote the natural projection of $\Omega$ on $S$ and the mapping whose existence and uniqueness are established in Proposition 1 . We refer to the structure thus obtained as the canonical preference structure:

$$
\mathscr{O}=\left(\Omega, \mathscr{A}, \varrho_{0}, \varpi\right) .
$$

This structure features three basic universality properties. Establishing these properties provides an answer to the question of which hierarchies are generated by some structure and what characterizes a set of hierarchies that can be thus obtained.

First, as our next result shows, furthering the claim in Proposition 1, the function $\varpi$ is onto. This means that every hierarchy is generated by some structure. The result indeed says much more: it states that the mapping from $\Omega$ to $S \times \Pi^{I}(\Omega)$ such that

\footnotetext{
${ }^{12}$ The intersection of an arbitrary family of algebras satisfying (1) and (2) is again an algebra with these properties, so our definitions are indeed meaningful.
} 
$\omega \mapsto\left(\varrho_{0}(\omega), \varpi(\omega)\right)$ not only preserves beliefs in the sense of Proposition 1 , but is in fact measurable and possesses a measurable inverse; we write all this using a more suggestive notation, as follows.

THEOREM 1. $\Omega \cong S \times \Pi^{I}(\Omega)$.

Second, given any preference structure $\mathscr{X}=(X, \mathscr{B}, \sigma, \vartheta)$, there is a unique morphism from $\mathscr{X}$ to $\mathscr{O},{ }^{13}$ namely, the generator of $\mathscr{X}$. Indeed, denoting the latter by $\gamma$, we have both $\varrho_{0} \circ \gamma=\sigma$ and

$$
\widehat{\gamma} \circ \vartheta^{i}=\varpi^{i} \circ \gamma \quad \forall i \in I,
$$

and it is immediate to see that $\gamma$ is the only mapping of $X$ into $\Omega$ with these properties.

Third, as the next two propositions show, every minimal and non-redundant structure can be identified with its generated set of hierarchies, and conversely every set of hierarchies that can be seen as a structure in its own right is minimal and non-redundant when so viewed. In order to state the results formally, we need the following definition.

Definition 4. A canonical substructure is a preference structure $\mathscr{O}^{\prime}=\left(\Omega^{\prime}, \mathscr{A}^{\prime}, \varrho_{0}^{\prime}, \varpi^{\prime}\right)$ such that $\left(\Omega^{\prime}, \mathscr{A}^{\prime}\right)$ is a subspace of $(\Omega, \mathscr{A})$ and the inclusion mapping of $\Omega^{\prime}$ into $\Omega$ is the morphism from $\mathscr{O}^{\prime}$ to $\mathscr{O}$. Since in this case $\mathscr{O}^{\prime}$ is uniquely determined by $\Omega^{\prime}$, we call $\mathscr{O}^{\prime}$ the canonical substructure induced by the set of hierarchies $\Omega^{\prime}$.

Proposition 3. Fix a set of hierarchies $\Omega^{\prime} \subseteq \Omega$. The following statements are equivalent.

(i) The set $\Omega^{\prime}$ induces a canonical substructure.

(ii) There exists a preference structure that generates $\Omega^{\prime}$.

(iii) Every $A \in \mathscr{A}$ with $\Omega^{\prime} \cap A=\varnothing$ is $\varpi^{i}(\omega)$-null for each player $i$ and each $\omega \in \Omega^{\prime}$.

The equivalence between (i) and (ii) in the proposition is further strengthened under minimality and non-redundancy.

Proposition 4. A preference structure is minimal and non-redundant if and only if it is isomorphic to the canonical substructure induced by its generated set of hierarchies.

As anticipated above, an immediate consequence of the latter result is that two minimal and non-redundant structures generating the same set of hierarchies $\Omega^{\prime}$ are isomorphic. In fact, they are both isomorphic to the same canonical substructure, and by Proposition 3 this canonical substructure is induced precisely by $\Omega^{\prime}$. Thus, a minimal and non-redundant structure can be interpreted as a set of hierarchies that is in some sense autonomous; the equivalence between (i) and (iii) in Proposition 3 indeed suggests that a set of hierarchies that induces a canonical substructure, like a beliefs subspace in Mertens and Zamir (1985), is characterized by the property that, whenever it obtains, all players are certain that it obtains-at least in the sense that, according to their preferences, all events disjoint from it are null.

\footnotetext{
${ }^{13}$ In other words, the canonical preference structure is universal in the sense of Heifetz and Samet (1998).
} 
In a related vein, a structure that generates a strict subset $\Omega^{\prime}$ of $\Omega$ embodies some form of restriction on the primitives $S$ or on preferences; either the projection of $\Omega^{\prime}$ on $\Omega_{n}$ is not onto for some $n$, so that some states of nature, or strategies, or certain preferences on $F\left(\Omega_{n-1}\right)$, never occur, ${ }^{14}$ or the projection on $\Omega_{n}$ is onto for all $n$, but still certain preferences on $F(\Omega, \mathscr{A})$ are ruled out. ${ }^{15}$ In any case, since only hierarchies satisfying such restrictions appear in $\Omega^{\prime}$, one would like to interpret the structure as a model where not only are the restrictions true, but each player is certain of this, is certain that all players are certain, and so on.

These interpretations are essentially correct. To investigate the precise extent to which they are, we must establish a link between the explicit restrictions-formulated within the canonical structure using a formal notion of certainty-and the preference structures where those restrictions are only implicitly assumed. This is indeed our task in the following two sections, where we provide an answer to our third main question above, i.e. we explain in what sense a structure can be seen as a set of hierarchies where some events obtain and this is common certainty. As we should expect given what we know about beliefs hierarchies, and even more so given our much less restricted preferences, this identification does not always hold, and characterizing those situations where it does raises a few delicate issues. Thus, before proceeding, it is useful to clarify these issues, paralleling them with the analogous, well-known ones regarding beliefs hierarchies, and draw a roadmap to the results in those sections.

\subsection{Common certainty and common belief: preliminaries}

The objective of our analysis in Sections 4 and 5 below is to give a qualification to the following statements. A set of hierarchies of the form $E \cap C C(E)$, where $E \subseteq \Omega$ and $C C(E)$ is the set of states where $E$ is common certainty, induces a canonical substructure. Conversely, a (necessarily minimal and non-redundant) preference structure is isomorphic to the canonical substructure induced by a set of hierarchies of that form.

For the first direction, the difficulty is with the definition of common certainty itself, since this forces us to consider events that are not in $\mathscr{A}$. Following Savage, we define certainty via (complements of) null events, but confining this definition to events in $\mathscr{A}$ is restrictive, as we also want or need to define certainty for such sets $E$ as, for instance, the set where Savage's axioms hold, the one where preferences are complete, and the one where a player is certain of some $A \in \mathscr{A}$, none of which belongs to $\mathscr{A}$. This apparent loss of generality entailed by our choice of $\mathscr{A}$, in turn dictated by the universality results of Section 3 (these would not hold under a richer class of events) would prove not too substantial if we could show that preferences on $F(\Omega, \mathscr{A})$ uniquely extend to acts measurable with respect to a larger family-one that is at least as large as $\mathscr{A}$ and contains

\footnotetext{
${ }^{14}$ For instance, it is immediate to see that if $\Omega^{\prime}$ is generated by a structure $(X, \mathscr{B}, \sigma, \vartheta)$ where $\vartheta^{i}(x)$ is complete for every $i \in I$ and every $x \in X$, then $\varpi^{i}(\omega)$ is also complete for all $i \in I$ and all $\omega \in \Omega^{\prime}$.

${ }^{15}$ This occurs, for instance, when one removes a single point from $\Omega$. (More generally, it occurs when we remove a subset of $\Omega$ that does not contain any event in $\mathscr{A}$.) Pick any $\omega \in \Omega$ and let $\Omega^{\prime}=\Omega \backslash\{\omega\}$. Then $\Omega^{\prime}$ is a strict subset of $\Omega$, but $A \cap \Omega^{\prime} \neq \varnothing$ for all $A \in \mathscr{A}$. (Indeed, $\{\omega\}$ is a singleton, while each $A \in \mathscr{A}$ is uncountable-see Lemma 5 in Appendix B.) Thus, $\Omega^{\prime}$ trivially satisfies (iii) in Proposition 3 , hence, by the equivalence between (ii) and (iii) in that proposition, it is generated by some structure.
} 
both the set $E$ of interest and the set where the players are certain of $E$ according to such extended preferences. This is indeed what Propositions 5 and 7 below do, allowing us to meaningfully define $E \cap C C(E)$ in two (mutually non-exclusive) cases: the first is when $E$ is an intersection of events in $\mathscr{A}$; the second is when $E$ is in $\mathscr{A}^{*}$, the $\sigma$-algebra generated by $\mathscr{A}$, and $\varpi^{i}(\omega)$ satisfies Savage's axioms for every $i \in I$ and $\omega \in E .{ }^{16}$ Note that one faces the analogous issue when defining beliefs about beliefs, i.e. one can only consider uncertainty spaces $X$ such that, for each measurable $E \subseteq X$, the set of probability measures on $X$ that put probability one on $E$ is measurable. ${ }^{17}$

For the second direction, the problem is identifying those properties of a structure that, while not themselves referring to properties of the generated set, make the structure isomorphic to (the canonical substructure induced by) some subset of $\Omega$ that has the form $E \cap C C(E)$ and is well defined in the sense above-i.e. where $E$ is either an intersection of events in $\mathscr{A}$, or an event in $\mathscr{A}^{*}$ where all players' preferences are Savage, or both. For the first case, the characterizing property is that the structure be nonredundant and simple, i.e. such that its events form a countable base for a compact Hausdorff topology. (This property is much more natural and easier to visualize than it might appear at first glance-see Section 6.3.) Indeed, Theorem 2 shows that, starting from an intersection $E$ of events in $\mathscr{A}$, the set $E \cap C C(E)$ induces a simple canonical substructure, and conversely every simple and non-redundant structure is isomorphic to the canonical substructure induced by some such set-in particular, it is automatically minimal. (Compare this to Mertens and Zamir's analysis, where only non-redundant and compact types spaces are considered, as these are, in the absence of metrizability, the only ones isomorphic to a well-defined beliefs subspace.) The second case is more complicated-hence our treatment in a separate section, because not even assuming a minimal and non-redundant structure $\mathscr{X}=(X, \mathscr{B}, \sigma, \vartheta)$ where each $\vartheta^{i}(x)$ is Savage is enough to deliver an element of $\mathscr{A}^{*}$ as its generated set. (In other words, there is no natural equivalent of "simple" in this case.) Instead, the characterizing property is that $\mathscr{X}$ be non-redundant and standard Borel, i.e. such that $\mathscr{B}$ is the $\sigma$-algebra generated by a Polish topology on $X$, and that $\vartheta^{i}(x)$ be Savage for all $i \in I$ and $x \in X$. Indeed, while such a structure is not itself isomorphic to a canonical substructure, we prove that its associated minimal structure is, and this is perfectly acceptable because from the latter one can recover $\mathscr{X}$ unambiguously and quite easily (note that here, too, minimality obtains as a result, not as an assumption.) (Once again, compare this to the beliefs hierarchies literature: in Brandenburger and Dekel's framework, one can embed only standard Borel type spaces into the universal type space, since only for such spaces is one guaranteed to get a measurable set of generated beliefs hierarchies.)

\footnotetext{
${ }^{16}$ Of course, this leaves open the possibility of other cases where unique extended preferences exist.

${ }^{17}$ In Mertens and Zamir's framework, where the primitive uncertainty space $S$ is assumed compact (not necessarily metrizable), the issue is resolved (i) using the fact that if $X$ is compact then $\Delta(X)$, the set of all probability measures on $X$ endowed with the weak* topology, is also compact, and (ii) considering only closed sets $E$. In Brandenburger and Dekel's framework, where $S$ is assumed Polish, no problem arises because of metrizability. (If $X$ is metrizable then so is $\Delta(X)$, and moreover the set $\{\mu \in \Delta(X): \mu(E)=1\}$ is measurable if $E$ is measurable—see e.g. Lemma 14.16 in Aliprantis and Border 1999.)
} 


\section{COMMON CERTAINTY AND SIMPLE STRUCTURES}

In this section we investigate sets of hierarchies that (i) induce a canonical substructure and (ii) can be written as an intersection of events in $\mathscr{A}$. We prove that such sets of hierarchies, in turn, arise naturally when we impose common certainty of an arbitrary set of hierarchies satisfying (ii). Finally, we introduce simple structures and prove that, under non-redundancy, these are precisely the ones isomorphic to the canonical substructures induced by the sets of hierarchies arising from common certainty.

\subsection{Certainty, common certainty, and closed components}

If a preference relation on $F(\Omega, \mathscr{A})$ is such that each event in a sequence from $\mathscr{A}$ is null, then it is natural to think of the union of the events in the sequence as a null event, too, even though it may not belong to $\mathscr{A}$. The following proposition formalizes this, showing that our intuition is also technically correct once we appeal to monotone continuity, an axiom that, as the result also shows, is automatically (in fact, trivially) satisfied by every preference relation on $F(\Omega, \mathscr{A})$. (We report the axiom in Appendix D since it is not important to do so here.)

Proposition 5. Every $\pi \in \Pi(\Omega, \mathscr{A})$ is monotone continuous. Let $\pi \in \Pi(\Omega, \mathscr{A})$ and let $E_{1}, E_{2}, \ldots$ be $\pi$-null events in $\mathscr{A}$. Let $E=\cup_{n} E_{n}$ and let $\mathscr{A}^{+}$be the algebra generated by $\mathscr{A} \cup\{E\}$. There exists a unique monotone continuous $\pi^{+} \in \Pi\left(X, \mathscr{A}^{+}\right)$such that

$$
\pi^{+} \cap[F(\Omega, \mathscr{A}) \times F(\Omega, \mathscr{A})]=\pi .
$$

Moreover, if the union $E^{\prime}$ of any sequence of events $E_{1}^{\prime}, E_{2}^{\prime}, \ldots$ in $\mathscr{A}$ satisfies $E^{\prime} \in \mathscr{A}^{+}$, then $E^{\prime}$ is $\pi^{+}$-null if and only if $E_{1}^{\prime}, E_{2}^{\prime}, \ldots$ are all $\pi$-null. In particular, $E$ is $\pi^{+}$-null.

Say a set of hierarchies $E \subseteq \Omega$ is closed if it is the intersection of a sequence of events $E_{1}, E_{2}, \ldots$ in $\mathscr{A} .{ }^{18}$ Based on the latter result, for every $\omega \in \Omega$, we say player $i$ is certain of $E$ at $\omega$, provided that $\Omega \backslash E_{1}, \Omega \backslash E_{2}, \ldots$ are all null according to $\varpi^{i}(\omega)$, and we define

$$
C^{i}(E)=\{\omega \in \Omega \text { : player } i \text { is certain of } E \text { at } \omega\} .
$$

The latter is itself a closed set of hierarchies, ${ }^{19}$ so for every $\omega \in \Omega$ we can define recursively: $E$ is 1-mutually certain at $\omega$ if every player is certain of $E$ at $\omega ; E$ is $(m+1)$ mutually certain at $\omega$ if $E$ is $m$-mutually certain at $\omega$ and, moreover, at $\omega$ every player is certain of

$$
M C_{m}(E)=\left\{\omega^{\prime} \in \Omega: E \text { is } m \text {-mutually certain at } \omega^{\prime}\right\}
$$

\footnotetext{
${ }^{18}$ This nomenclature is justified by the fact that the family of intersections of events in $\mathscr{A}$ is precisely the family of subsets of $\Omega$ that are closed in the topology generated by $\mathscr{A}$. This becomes evident when we introduce the notion of simple space below.

${ }^{19}$ By Proposition 5, certainty of $E$ is well defined, i.e. does not depend on the particular sequence $E_{1}, E_{2}, \ldots$. Moreover, $C^{i}(E)$ is itself closed because $F(\Omega, \mathscr{A})$ is countable and $C^{i}(E)$ can be written as the intersection of all sets of the form $\left\{\omega \in \Omega:\left(h E_{n} f, h E_{n} g\right) \in \varpi^{i}(\omega)\right\}$, where $n \geq 1$ and $f, g, h \in F(\Omega, \mathscr{A})$.
} 
finally, $E$ is commonly certain at $\omega$ if $E$ is $m$-mutually certain at $\omega$ for all $m$. Observe that, since $M C_{m}(E)$ is closed for every $m$, so is

$$
C C(E)=\{\omega \in \Omega: E \text { is commonly certain at } \omega\} .
$$

A subset of $\Omega$ having the form $E \cap C C(E)$, where $E$ is closed, is called a closed component. The next proposition formalizes and delineates the scope of our earlier informal arguments: canonical substructures induced by closed sets of hierarchies are characterized by the property of being a certainty among players whenever they obtain; moreover, they can be thought of as closed components, i.e. they correspond to situations where certain restrictions on preferences (some closed set $E$ ) hold and this is common certainty.

Proposition 6. For every closed $\Omega^{\prime} \subseteq \Omega$ the following statements are equivalent.

(i) $\Omega^{\prime}$ induces a canonical substructure.

(ii) $\Omega^{\prime} \subseteq M C_{1}\left(\Omega^{\prime}\right)$

(iii) $\Omega^{\prime}$ is a closed component.

\subsection{Simple structures}

The equivalence between (i) and (iii) in Proposition 6 allows us to turn the question of how a preference structure can be seen as a situation where certain events hold, and this is common certainty, into the following: What properties of a preference structure, beyond minimality and non-redundancy, make it isomorphic to a canonical substructure induced by a closed set of hierarchies?

Definition 5. A space $(X, \mathscr{B})$ is simple if $\mathscr{B}$ is a countable base for a compact Hausdorff topology on $X$. A structure $\mathscr{X}=(X, \mathscr{B}, \sigma, \vartheta)$ is simple if the space $(X, \mathscr{B})$ is simple.

The next theorem shows that the definition above indeed provides the right notion. Note the following two immediate corollaries of the result. First, the canonical structure is itself simple-which we in fact prove directly in Appendix B. Second, every simple and non-redundant structure is minimal.

THEOREM 2. A preference structure is simple and non-redundant if and only if it isomorphic to the canonical substructure induced by a closed component.

\section{COMmon Belief AND STANDARd Savage STRUCtURES}

In this section we introduce Savage preferences and analyze the sets of hierarchies that (i) induce a canonical substructure, (ii) belong to the $\sigma$-algebra $\mathscr{A}^{*}$ generated by $\mathscr{A}$, and (iii) feature Savage's axioms everywhere. We prove that such sets of hierarchies arise when we impose both Savage's axioms and common certainty (hence common belief) of a set of hierarchies satisfying (ii) and (iii). We introduce standard Savage structures and 
prove that, under non-redundancy, their associated minimal structures, which characterize them completely and explicitly, are precisely the ones isomorphic to the canonical substructures arising from common belief of some event in $\mathscr{A}^{*}$. Finally, we prove that type spaces like those commonly used in the economics literature are just equivalent formulations of standard Savage structures, thus concluding the analysis.

\subsection{Belief, common belief, and Savage components}

Given a space $(X, \mathscr{B})$, we say a preference relation $\pi \in \Pi(X, \mathscr{B})$ is Savage if it satisfies axioms P1-P6 in Savage (1954). (The axioms are well known and we do not need to list them here, so we relegate them to Appendix D.) By Kopylov's (2007) generalization of Savage's theorem to algebras, ${ }^{20}$ this is equivalent to the following. There exist a utility function, i.e. a mapping $u: Z \rightarrow[0,1]$ with $\max _{z} u(z)=1$ and $\min _{z} u(z)=0$, and a finely ranged belief, i.e. a finitely additive $\mu: \mathscr{B} \rightarrow[0,1]$ with $\mu(X)=1$ and $\{\mu(B): A \supseteq B \in \mathscr{B}\}$ dense in $[0, \mu(A)]$ for all $A \in \mathscr{B}$, satisfying, for all $f, g \in F(X, \mathscr{B})$,

$$
(f, g) \in \pi \quad \text { if and only if } \sum_{z \in Z} u(z) \mu\left(f^{-1}(z)\right) \geq \sum_{z \in Z} u(z) \mu\left(g^{-1}(z)\right) .
$$

If such a pair $(u, \mu)$ exists, it is unique, and we call it the SEU representation of $\pi$; moreover, in this case, $\mu$ is countably additive if and only if $\pi$ is monotone continuous. ${ }^{21}$

Now let $\mathscr{A}^{*}$ denote the family of Borel subsets of $\Omega$, i.e. the $\sigma$-algebra generated by $A^{22}$ We define belief, i.e. certainty under Savage preferences, for every Borel $E \subseteq \Omega$, based on the following result.

Proposition 7. Let $(X, \mathscr{B})$ be a space, let $\pi \in \Pi(X, \mathscr{B})$, and let $\mathscr{B} *$ be the $\sigma$-algebra generated by $\mathscr{B}$. Then $\pi$ is monotone continuous and Savage if and only if there exists a monotone continuous and Savage $\pi^{*} \in \Pi\left(X, \mathscr{B}^{*}\right)$ such that

$$
\pi^{*} \cap[F(X, \mathscr{B}) \times F(X, \mathscr{B})]=\pi .
$$

In this case, $\pi^{*}$ is unique, and every event in $\mathscr{B}$ is $\pi$-null if and only if it is $\pi^{*}$-null.

Indeed, we know from Proposition 5 that every preference relation on $F(\Omega, \mathscr{A})$ is monotone continuous. Thus, for every $E \in \mathscr{A}^{*}$, we say player $i$ believes $E$ at $\omega \in \Omega$ if

\footnotetext{
${ }^{20}$ In this paper we do not investigate alternative axiomatics for expected utility. In particular, since we assume finitely many outcomes, e.g. Gul's (1992) result is not applicable here, and to get uniqueness of the SEU representation we have to resort to non-atomicity, which is implied by Savage's axiom P6.

${ }^{21}$ If $\pi$ is monotone continuous and $\mathscr{B}$ is in fact a $\sigma$-algebra then we can-in addition, but in fact, by Lyapunov's theorem (see e.g. Lindenstrauss 1966), equivalently-require $\mu$ to be convex ranged, i.e. $\{\mu(B)$ : $A \supseteq B \in \mathscr{B}\}=[0, \mu(A)]$ for all $A \in \mathscr{B}$, or, still equivalently, non-atomic, i.e. $\{\mu(B): A \supseteq B \in \mathscr{B}\} \neq\{0, \mu(A)\}$ for all $A \in \mathscr{B}$ with $\mu(A)>0$. The equivalence (in the presence of P1-P6) between countable additivity of $\mu$ and monotone continuity of $\pi$ has been proved in Villegas (1964) and Arrow (1970) for the case where $\mathscr{B}$ is a $\sigma$-algebra. Their proofs taken verbatim are valid even if $\mathscr{B}$ is assumed to be an algebra.

${ }^{22}$ As we prove in Appendix B, the space $(\Omega, \mathscr{A})$ is simple, hence $\mathscr{A}^{*}$ is indeed the Borel $\sigma$-algebra generated by the topology generated by $\mathscr{A}$. In general, for any space $(X, \mathscr{B})$ where $\mathscr{B}$ is countable, the topology generated by $\mathscr{B}$ generates the same $\sigma$-algebra as $\mathscr{B}$ itself.
} 
the relation $\varpi^{i}(x)$ is Savage and if, moreover, $\Omega \backslash E$ is null according to the extension of $\varpi^{i}(x)$ given by Proposition $7 .{ }^{23}$ Now define

$$
C_{\mathrm{SAV}}^{i}(E)=\{\omega \in \Omega \text { : player } i \text { believes } E \text { at } \omega\}
$$

and note that $C_{\mathrm{SAV}}^{i}(\Omega)$ is the set all $\omega \in \Omega$ such that $\varpi^{i}(\omega)$ is Savage. Then we have the following result.

Proposition 8. $C_{\mathrm{SAV}}^{i}(E) \in \mathscr{A}^{*}$ for every $E \in \mathscr{A}^{*}$ and every player $i$.

Thus, for every Borel set of hierarchies $E \in \mathscr{A}^{*}$ and every $\omega \in \Omega$, we can define: $E$ is 1-mutual belief at $\omega$ if every player believes $E$ at $\omega$; recursively for all $m \geq 1, E$ is $(m+1)$-mutual belief at $\omega$ if $E$ is $m$-mutual belief at $\omega$ and, moreover, at $\omega$ every player believes

$$
M C_{\mathrm{SAV}, m}(E)=\left\{\omega^{\prime} \in \Omega: E \text { is } m \text {-mutual belief at } \omega^{\prime}\right\}
$$

finally, $E$ is common belief at $\omega$ if $E$ is $m$-mutual belief at $\omega$ for all $m$, and we let

$$
C C_{\mathrm{SAV}}(E)=\{\omega \in \Omega: E \text { is common belief at } \omega\} .
$$

A Savage component is a subset of $\Omega$ having the form $E \cap C C_{\mathrm{SAV}}(E)$ for some $E \in \mathscr{A}^{*}$. Savage components are obviously Borel sets themselves, and satisfy properties analogous to those established in Proposition 6 for closed components. According to the next result, canonical substructures induced by Borel sets of hierarchies where Savage's axioms hold everywhere are characterized by the property of being believed whenever they obtain; moreover, these sets of hierarchies are precisely the Savage components, i.e. correspond to situations where certain restrictions on preferences-namely, Savage's axioms, plus some Borel set $E$-hold, and this is common belief.

Proposition 9. For every $E \in \mathscr{A}^{*}$ the following statements are equivalent.

(i) E is a Savage component.

(ii) $E \subseteq M C_{\mathrm{SAV}, 1}(E)$.

(iii) E induces a canonical substructure, and $E \subseteq M C_{\mathrm{SAV}, 1}(\Omega)$.

\subsection{Standard Savage structures and type spaces}

Paralleling our analysis of closed components and simple structures, here we ask what properties of a preference structure allow us to interpret it as a situation where Savage's axioms (and possibly some other restriction, i.e. some Borel set $E \subseteq \Omega$ ) hold and there is common certainty of this. By the equivalence between (i) and (iii) in Proposition 9, this question can be rephrased thus: Given a structure $\mathscr{X}=(X, \mathscr{B}, \sigma, \vartheta)$, what properties of the space $(X, \mathscr{B})$ and of the mapping $\vartheta: X \rightarrow \Pi^{I}(X, \mathscr{B})$ are necessary and sufficient to

\footnotetext{
${ }^{23}$ As an obvious consequence of Lemma 7 in Appendix C, the notions of belief and certainty-as defined earlier-indeed agree. More precisely, $C_{\mathrm{SAV}}^{i}(E)=C_{\mathrm{SAV}}^{i}(\Omega) \cap C^{i}(E)$ for every closed $E \subseteq \Omega$.
} 
make $\mathscr{X}$ isomorphic to the canonical substructure induced by some $E \in \mathscr{A}^{*}$ satisfying $E \subseteq M C_{\mathrm{SAV}, 1}(\Omega)$ ? The following definition provides the answer.

Recall that a standard Borel space is a space $(X, \mathscr{B})$ where $\mathscr{B}$ is the Borel $\sigma$-algebra generated by a Polish topology on $X$-see, for instance, Kechris (1995).

Definition 6. A preference structure $(X, \mathscr{B}, \sigma, \vartheta)$ is Savage if $\vartheta^{i}(x)$ is Savage for every $i \in I$ and every $x \in X$. It is standard Savage if, in addition, $(X, \mathscr{B})$ is a standard Borel space and $\vartheta^{i}(x)$ is monotone continuous for every $i \in I$ and every $x \in X$.

The key observation is that the standard Savage structures are all and only those structures that can be uniquely obtained starting from their associated minimal structures and considering the generated $\sigma$-algebras of events, as in the second part of the following result.

Proposition 10. Let $(X, \mathscr{B}, \sigma, \vartheta)$ be a non-redundant standard Savage structure. Then its associated minimal structure $(X, \underline{\mathscr{B}}, \sigma, \underline{\vartheta})$ is non-redundant Savage, and $\underline{\mathscr{B}}$ generates $\mathscr{B}$.

Indeed, the minimal structures associated with non-redundant standard Savage structures are, in turn, precisely those isomorphic to the canonical substructures induced by the Savage components, as the main result of this section now shows.

THeOREM 3. A preference structure is isomorphic to the canonical substructure induced by a Savage component if and only if it is the minimal structure associated with a nonredundant standard Savage structure $\mathscr{X}$. Moreover, in this case, $\mathscr{X}$ is unique.

Note that the full force of the theorem, and in particular the uniqueness part, appears only in conjunction with Propositions 7 and 10, since these results add to the uniqueness of the standard Savage structure $\mathscr{X}$ by explaining exactly how to derive this structure. Indeed, starting from its associated minimal structure, say $(X, \underline{\mathscr{B}}, \sigma, \underline{\vartheta})$, all we have to do is compute the $\sigma$-algebra $\mathscr{B}$ generated by $\underline{\mathscr{B}}$ and define $\vartheta: X \rightarrow \Pi^{I}(X, \mathscr{B})$ via Proposition 7. Then Propositions 7 and 10 together guarantee that the tuple $(X, \mathscr{B}, \sigma, \vartheta)$ thus obtained is exactly the structure $\mathscr{X}$. Moreover, from the proof of Proposition 7 we know that describing the mapping $\vartheta$-and hence the structure $\mathscr{X}$ itself-involves a simple procedure: for each player $i$ and each $x \in X$ one takes the utility function and the belief on $\underline{\mathscr{B}}$ representing $\underline{\vartheta}^{i}(x)$, and then defines $\vartheta^{i}(x)$ as the Savage relation represented by the same utility function and by the same belief, extended to $\mathscr{B}$ as usual.

We conclude this section by showing that the commonly used tools for modeling asymmetric information situations in economics, i.e. type spaces à la Harsanyi, are completely equivalent to standard Savage structures, and hence also equivalent to Savage components, tying our conclusions together. Let $\mathscr{U}$ denote the set of all utility functions and, given a space $(X, \mathscr{B})$, let $\Delta_{\mathrm{F}}(X, \mathscr{B})$ be the set of all countably additive, finely ranged beliefs on $(X, \mathscr{B})$. Consistently with existing notation, let $\mathscr{U}^{I}=\times_{i \in I} \mathscr{U}$ and $\Delta_{\mathrm{F}}^{I}(X, \mathscr{B})=$ $\times_{i \in I} \Delta_{\mathrm{F}}(X, \mathscr{B})$ and, given another space $Y$ and a function $\phi$ from $Y$ to $\mathscr{U}^{I}$ or $\Delta_{\mathrm{F}}^{I}(X, \mathscr{B})$, write $\phi^{i}$ for the function mapping $y \in Y$ into the $i$ th coordinate of $\phi(y)$. 
Definition 7. A standard Savage type space is a tuple $\mathscr{T}=(X, \mathscr{B}, \sigma, v, \beta)$ where: $(X, \mathscr{B})$ is a standard Borel space; $\sigma: X \rightarrow S$ is measurable; and $v^{i}: X \rightarrow \mathscr{U}$ and $\beta^{i}: X \rightarrow \Delta_{\mathrm{F}}(X, \mathscr{B})$ satisfy

$$
\begin{array}{ll}
\left\{x \in X: v^{i}(x)[z]>u\right\} \in \mathscr{B} & \forall z \in Z, \forall u \in[0,1] \\
\left\{x \in X: \beta^{i}(x)[E]>p\right\} \in \mathscr{B} & \forall E \in \mathscr{B}, \forall p \in[0,1] .
\end{array}
$$

If the smallest $\sigma$-algebra for which both (5) and (6) hold separates every two distinct elements of $X$, we say $\mathscr{T}$ is non-redundant. ${ }^{24}$

A standard Savage type space $(X, \mathscr{B}, \sigma, v, \beta)$ induces a standard Savage structure $(X, \mathscr{B}, \sigma, \vartheta)$ in the obvious way, i.e. defining $\vartheta: X \rightarrow \Pi^{I}(X, \mathscr{B})$ by stipulating that, for every $i \in I$ and every $x \in X$, the preference relation $\vartheta^{i}(x)$ is the one represented by the utility function $v^{i}(x)$ and the belief $\beta^{i}(x)$. By (5) and (6), this mapping is measurable, so $(X, \mathscr{B}, \sigma, \vartheta)$ is indeed a standard Savage structure. According to the following result, the converse also holds, i.e. every standard Savage structure is induced by some standard Savage type space, hence the two notions are entirely equivalent.

Proposition 11. Let $\mathscr{X}$ be a standard Savage structure. There exists a unique standard Savage type space $\mathscr{T}$ inducing $\mathscr{X}$. Furthermore, $\mathscr{T}$ is non-redundant if and only if $\mathscr{X}$ is non-redundant.

\section{DisCUSSION AND EXTENSIONS}

\subsection{Preference structures in Epstein and Wang's framework}

As mentioned in the introduction, Epstein and Wang (1996) assume a compact Hausdorff basic uncertainty space. Thus, while in their paper preference structures are not formally defined, it is reasonable to conjecture that such a definition-along with isomorphism results analogous to our Theorems 2 and 3-can be provided, following Mertens and Zamir's analysis, i.e. for (non-redundant) preference structures whose underlying space is compact Hausdorff and all functions involved are continuous. Similarly, we conjecture that, in the particular case where the basic space of uncertainty is, in addition, Polish, one obtains an even better-behaved universal space in which all (non-redundant) standard Borel preference structures (be they Savage or not) can be embedded (in the measurable sense).

\footnotetext{
${ }^{24}$ Requirements (5) and (6) are equivalent to the measurability of $v^{i}$ and $\beta^{i}$ when $\mathscr{U}$ is seen as a subset of $[0,1]^{Z}$ endowed with the usual (relative) product Borel $\sigma$-algebra, and $\Delta_{\mathrm{F}}(X, \mathscr{B})$ is endowed with the $\sigma$ algebra generated by the sets of the form $\left\{\mu \in \Delta_{\mathrm{F}}(X, \mathscr{B}): \mu(E)>p\right\}$ where $E \in \mathscr{B}$ and $p \in[0,1]$. The latter $\sigma$-algebra is widely used in the literature on type spaces and, since we assume $(X, \mathscr{B})$ is standard Borel, coincides with the one generated by the topology of weak convergence of measures on that space. (See e.g. Theorem 17.24 in Kechris 1995.) Our definition of non-redundancy is taken from Mertens and Zamir (1985) and coincides with injectivity of the canonical mapping from $X$ to the universal S-based beliefs space constructed in that paper.
} 


\subsection{The basic events in a space of preferences}

Throughout the paper we assume that, given a space $(X, \mathscr{B})$, the set $\Pi(X, \mathscr{B})$ is equipped with the algebra generated by

$$
\{\{\pi \in \Pi(X, \mathscr{B}):(f, g) \in \pi\}: f, g \in F(X, \mathscr{B})\} .
$$

Although one may think of other options, ${ }^{25}$ we consider this as the right choice for at least two reasons - of course, besides the fact itself that it makes our results true. First, in the minimal case, and in general when $\mathscr{B}$ is countable, the set of acts $F(X, \mathscr{B})$ is countable. In this case, one can put the acts of $F(X, \mathscr{B}) \times F(X, \mathscr{B})$ in a sequence-e.g. listing them according to how "complicated" a pair $(f, g)$ is, say, how many distinct outcomes are involved, etc.- - and then a basic event simply specifies, for each of the first $n$ pairs of acts, whether the first is preferred to the second. In our opinion, this is quite natural. Second, and perhaps more importantly, in the case where $X$ is part of a Savage structure the family of events specified above is exactly the one that generates the usual $\sigma$-algebras on $\mathscr{U}$ and $\Delta_{\mathrm{F}}(X, \mathscr{B})$ when we pass to the associated standard Savage type space (see footnote 24). We take this as a further indication that our choice is appropriate, since it makes preference structures a true generalization of type spaces as we see them from the existing literature.

\subsection{Examples of simple spaces}

The definition of simple space is not really transparent, yet simple spaces are quite easy to visualize and occur extremely often in economic environments. The canonical example of an infinite simple space is the set $\{0,1\}^{\mathbb{N}}$ endowed with the algebra generated by the cylinders. Indeed, every simple space is homeomorphic to $\{0,1\}^{\mathbb{N}}$. In other words, simple spaces are those we can depict as (infinite) trees, with sets of points having the same $n$ initial branches as basic events. Thus, simple spaces are among the most natural mathematical objects an economist (and in particular a game theorist) can think of; one can see $\{0,1\}^{\mathbb{N}}$ coming from an infinite sequence of coin tosses, from the set of truth assignments to the formulas generated by a countable alphabet, from the infinite repetition of a finite stage game, and from many more standard examples. In each case, the events (or equivalently the clopen (closed and open) sets-see Appendix A) in the equipped algebra have a very clear interpretation: they are finite unions of statements like "the first 4 tosses were $H, T, T, T$ ", "formulas 1,2 are true, formula 5 is false", "player $i$ chose 'left' in the first 3 stages and 'right' in the fourth stage", etc.

\footnotetext{
${ }^{25}$ Since $\Pi\left(\Omega_{n}\right)$ is finite for every $n$, there is really no choice at the lower orders, so the only question is how our results would change if a family of events other than the algebra generated by the sets of the form $\{\pi \in \Pi(\Omega, \mathscr{A}):(f, g) \in \pi\}$ was assumed on $\Pi(\Omega, \mathscr{A})$. Given our choice of $\mathscr{A}$, the answer to that question is that the universality results in Section 3 would not hold under any other class of events. In particular, any larger class would make the crucial, second part of Proposition 1 (hence also Theorem 1) fail, whereas any smaller class would not even contain enough information to describe lower order preferences. This is because, as we see from the proof of Proposition 1 in Appendix B, every act in $F(\Omega, \mathscr{A})$ can be written as $f \circ \varrho_{n}$ for some $n$ and some $f \in F\left(\Omega_{n}\right)$. It is this property, which crucially depends on the finiteness of $Z$, that allows us to avoid the usual difficulties involved in going to the limit. (Contrast this to Epstein and Wang's 1996 model, where regularity assumptions are needed.)
} 


\subsection{Rationality}

In order to see a strategy of a player as an act in $F(S)$, one must first specify an outcome function, i.e. a mapping $\zeta: S \rightarrow Z$. Although this plays no role in our analysis, it is also necessary in order to discuss rationality. For every player $i$, identify each $s^{i} \in S^{i}$ with the act mapping $S$ into $Z$ such that $\left(\widetilde{s}^{i}, s^{-i}\right) \mapsto \zeta\left(s^{i}, s^{-i}\right)$ for every $\widetilde{s}^{i} \in S^{i}$ and every $s^{-i} \in S^{-i}$. Player $i$ is rational at $\omega \in \Omega$ if there does not exist $s^{i} \in S^{i}$ such that $\left(s^{i}, \varrho_{0}^{i}(\omega)\right) \dot{\in} \varpi^{i}(\omega)$. It is clear that the set

$$
R^{i}=\{\omega \in \Omega: \text { player } i \text { is rational at } \omega\}
$$

is closed. Thus, $R=\cap_{i \in I} R^{i}$ is also closed, therefore $\Omega_{\mathrm{RAT}}=R \cap C Q(R)$ is the closed component where all players are rational and this is common certainty.

\subsection{The canonical standard Savage structure}

Let $\Omega_{\mathrm{SAV}}=C C_{\mathrm{SAV}}(\Omega)$. This is the subset of $\Omega$ where all players' preferences are Savage and this is common belief. Let $\mathscr{A}_{\mathrm{SAV}}$ and $\mathscr{A}_{\mathrm{SAV}}^{*}$ denote the relative algebra and $\sigma$-algebra on $\Omega_{\mathrm{S}}$, respectively. Then the set

$$
\Omega_{\mathrm{SAV}}=M C_{\mathrm{SAV}, 1}(\Omega) \cap C C_{\mathrm{SAV}}\left(M C_{\mathrm{SAV}, 1}(\Omega)\right)
$$

is a Savage component and hence induces a Savage canonical substructure. The latter is

$$
\left(\Omega_{\mathrm{SAV}}, \mathscr{A}_{\mathrm{SAV}}, \varrho_{0, \mathrm{SAV}}, \varpi_{\mathrm{SAV}}\right)
$$

where $\varrho_{0, \mathrm{SAV}}: \Omega_{\mathrm{SAV}} \rightarrow S$ restricts $\varrho_{0}$ to $\Omega_{\mathrm{SAV}}$ and $\varpi_{\mathrm{SAV}}^{i}: \Omega_{\mathrm{SAV}} \rightarrow \Pi\left(\Omega_{\mathrm{SAV}}, \mathscr{A}_{\mathrm{SAV}}\right)$ maps

$$
\omega \mapsto\left\{(f \circ \iota, g \circ \iota):(f, g) \in \varpi^{i}(\omega)\right\},
$$

where $\iota: \Omega_{\mathrm{SAV}} \rightarrow \Omega$ is the inclusion mapping. By Propositions 7 and 10 , this structure in turn induces the following canonical standard Savage structure: ${ }^{26}$

$$
\mathscr{O}_{\mathrm{SAV}}^{*}=\left(\Omega_{\mathrm{SAV}}, \mathscr{A}_{\mathrm{SAV}}^{*}, \varrho_{0, \mathrm{SAV}}, \varpi_{\mathrm{SAV}}^{*}\right) \text {. }
$$

This structure features universality properties analogous to those satisfied by $\mathscr{O}$. Indeed, letting $\Pi_{\mathrm{SAV}}\left(\Omega_{\mathrm{SAV}}, \mathscr{A}_{\mathrm{SAV}}^{*}\right)$ denote the family of monotone continuous Savage preference relations on $F\left(\Omega_{\mathrm{SAV}}, \mathscr{A}_{\mathrm{SAV}}^{*}\right)$, the mapping of $\Omega_{\mathrm{SAV}}$ into $S \times \Pi_{\mathrm{SAV}}^{I}\left(\Omega_{\mathrm{SAV}}, \mathscr{A}_{\mathrm{SAV}}^{*}\right)$ such that $\omega \mapsto\left(\varrho_{0}(\omega), \varpi_{\text {SAV }}^{*}(\omega)\right)$ is measurable and possesses an inverse. ${ }^{27}$ Furthermore, the latter becomes measurable, provided that each copy of $\Pi_{\mathrm{SAV}}\left(\Omega_{\mathrm{SAV}}, \mathscr{A}_{\mathrm{SAV}}^{*}\right)$ is endowed with the $\sigma$-algebra generated by the sets

$$
\left\{\pi \in \Pi_{\mathrm{SAV}}\left(\Omega_{\mathrm{SAV}}, \mathscr{A}_{\mathrm{SAV}}^{*}\right):(f, g) \in \pi\right\}
$$

where $f, g \in F\left(\Omega_{\mathrm{SAV}}, \mathscr{A}_{\mathrm{SAV}}^{*}\right)$. Extending our earlier notation, we may write this as

$$
\left(\Omega_{\mathrm{SAV}}, \mathscr{A}_{\mathrm{SAV}}^{*}, \varrho_{0, \mathrm{SAV}}, \varpi_{\mathrm{SAV}}^{*}\right) \cong_{\mathrm{SAV}} S \times \Pi_{\mathrm{SAV}}^{I}\left(\Omega_{\mathrm{SAV}}, \mathscr{A}_{\mathrm{SAV}}^{*}\right) .
$$

Finally, one can define substructures of $\mathscr{O}_{\text {SAV }}$ just like we did for $\mathscr{O}$, and argue that every standard Savage structure can be seen as such a substructure. See Di Tillio (2006) for details.

\footnotetext{
${ }^{26}$ Here $\varpi_{\mathrm{SAV}}^{* i}: \Omega_{\mathrm{SAV}} \rightarrow \Pi\left(\Omega_{\mathrm{SAV}}, \mathscr{A}_{\mathrm{SAV}}^{*}\right)$ is the mapping induced by $\varpi_{\mathrm{SAV}}^{i}$ via Proposition 7 .

${ }^{27}$ The proof of this is immediate and we omit it here. It can be found in Di Tillio (2006).
} 


\subsection{Canonical structures for closed sets of axioms}

Choose an arbitrary set of axioms for a preference relation on $F(\Omega, \mathscr{A})$. Say this set of axioms is closed if the subset of $\Pi(\Omega, \mathscr{A})$ where the axioms are satisfied is closed. If we choose a closed set of axioms for each player (possibly different sets of axioms for different players), then our results guarantee that a suitably constructed closed component features both the axioms and common certainty of them. Let $\mathscr{O}_{\mathrm{A}}$ denote the induced substructure and say a preference structure $(X, \mathscr{B}, \sigma, \vartheta)$ is consistent with the chosen axioms if, for every player $i$ and all $x \in X$, the relation $\varpi^{i}(\gamma(x))$ induced on $F(\Omega, \mathscr{A})$ satisfies the set of axioms chosen for player $i$. (As usual, $\gamma$ denotes the generator.) One can define substructures of $\mathscr{O}_{\mathrm{A}}$ as we did for $\mathscr{O}$, and then conclude that every simple and non-redundant structure consistent with the chosen sets of axioms is isomorphic to a substructure of $\mathscr{O}_{\mathrm{A}}$. In other words, the latter is also universal, but relative to the chosen sets of axioms. See Di Tillio (2006) for details.

It is worth pointing out that all finite or countable sets of axioms involving only the quantifier "for all" applied to events, outcomes, acts, finite partitions of the space into events, etc. are closed. For example, all of Savage's axioms except P6 are closed-when each is seen as a set of axioms-and therefore any set of axioms comprising one or more of $\mathrm{P} 1, \ldots, \mathrm{P} 5$ is closed. Indeed, when dealing with structures where the family of events is countable, every axiom involving conditions of the form "for every event $A$ ", "for all acts $f$ and $g$ ", etc. can be written as a countable intersection of events. On the other hand, P6 does not have this form; indeed, it is a tail property, since it corresponds to a countable intersection of countable unions- "for every outcome and every pair of acts, there exists a finite partition such that...". As a result, there is no canonical substructure that is universal relative to $\mathrm{P} 6$ only, even though for all of $\mathrm{P} 1-\mathrm{P} 6$ the canonical standard Savage structure is universal in the sense discussed above.

\subsection{Own strategies and preferences}

It is easy to construct a closed component where (i) every player is certain of his own strategy and preferences, and (ii) there is common certainty of (i). It suffices to note that, for each player $i$, the set

$$
D^{i}=\left\{\omega \in \Omega \text { : player } i \text { is certain at } \omega \text { of }\left(\varrho_{0}^{i}\right)^{-1}\left(\varrho_{0}^{i}(\omega)\right) \cap\left(\varpi^{i}\right)^{-1}\left(\varpi^{i}(\omega)\right)\right\}
$$

is closed, hence $D=\cap_{i \in I} D^{i}$ is closed and $\Omega_{\mathrm{OWN}}=D \cap C C(D)$ is a closed component. Observe also that $\Omega_{\mathrm{SAV}} \cap \Omega_{\mathrm{OWN}}$ belongs to $\mathscr{A}^{*}$, therefore

$$
\Omega_{\mathrm{SAV}, \mathrm{OWN}}=\left(\Omega_{\mathrm{SAV}} \cap \Omega_{\mathrm{OWN}}\right) \cap C C_{\mathrm{SAV}}\left(\Omega_{\mathrm{SAV}} \cap \Omega_{\mathrm{OWN}}\right)
$$

is the Savage component where all players' preferences satisfy P1-P6, all players are certain of their own strategies and preferences, and this is common belief.

\subsection{Complete information and subjective correlated equilibrium}

Fix a utility function $u^{i}: Z \rightarrow \mathbb{R}$ for every player $i$. By Lemma 11 in Appendix $\mathrm{E}$, the set

$$
D=\left\{\omega \in \Omega_{\mathrm{SAV}, \mathrm{OWN}}: \forall i \exists \mu^{i} \in \Delta_{\mathrm{F}}(\Omega, \mathscr{A}) \text { s.t. }\left(u^{i}, \mu^{i}\right) \text { is the SEU representation of } \varpi^{i}(\omega)\right\},
$$


where $\Omega_{\mathrm{SAV}, \mathrm{OWN}}$ is as above, belongs to $\mathscr{A}^{*}$. Thus, $\Omega_{\mathrm{SAV}, \mathrm{OWN}, \mathrm{C}}=D \cap C C_{\mathrm{SAV}}(D)$ is a $\operatorname{Sav}$ age component, hence it induces a Savage canonical substructure. This has a unique associated standard Savage structure and hence a unique associated standard Savage type space. The latter features complete information, i.e. the players may be uncertain about other players' beliefs, but are certain of their own strategies, beliefs, and utilities, certain of the other players' utilities, and commonly certain of these certainties. This type space is essentially identical to the mathematical object assumed in the original definition of (a posteriori) subjective correlated equilibrium; in particular, Aumann (1974) explicitly imposes non-atomic beliefs, which in Savage's context (P6) and ours is precisely what guarantees existence and uniqueness of beliefs. While without a doubt an interesting exercise, a full exploration of the precise connections between Aumann's (and also Forges's 1993) theory and ours goes beyond the scope of this paper.

\subsection{Finite order preferences and finite order beliefs}

In Mertens and Zamir (1985) the basic uncertainty is represented by a compact space, which can be interpreted as the set of all possible states of nature and payoff functions. Now for every $n \geq 1$ our space $\Omega_{n}$ is finite, whereas the cardinality of the lower order spaces is already very high in Mertens and Zamir's construction, and this would be so even if their basic uncertainty space were assumed to be finite. Since pairs of utility functions and beliefs correspond to a particular kind of preference relations, it might be argued, on the contrary, that the spaces constructed in this paper should be larger. In other words, it is natural to ask whether we are really missing something relevant here.

The answer is a qualified "no." The reason for our simpler construction is that only a finite, but exhaustive family of sets of pairs of utility functions and beliefs appear at each level. This depends, of course, on the fact that each $\Omega_{n}$ is finite, hence no preference relation on $F\left(\Omega_{n}\right)$ can have a unique SEU representation. This ambiguity, however, disappears when we consider an entire preference hierarchy. By Savage's theorem, for any player $i$ and at any $\omega \in \Omega_{\mathrm{SAV}}$ a unique SEU representation of $\varpi^{i}(\omega)$ exists, and the belief in this SEU representation pins down exactly the $n$-order belief of player $i$ for all $n \geq 1$. Moreover, every $n$-order belief can be generated this way. The qualification to our answer, of course, is that the belief in the SEU representation of $\varpi^{i}(\omega)$ must be finely ranged, hence, for instance, our model rules out finite or countable type spaces.

Let us illustrate this using an example with two outcomes, $Z=\{b, g\}$, two players, $I=\{1,2\}$, two strategies for each player, $S^{1}=\{l, r\}$ and $S^{2}=\{\mathrm{u}, \mathrm{d}\}$, and one state of nature, $S^{0}=\{\mathrm{s}\}$. Consider the standard Savage type space $(X, \mathscr{B}, \sigma, v, \beta)$ defined as follows. Let $X^{1}=X^{2}=[0,1]$, let $X=X^{1} \times X^{2}$, and let $\mathscr{B}$ be the usual Borel $\sigma$-algebra. Both players strictly prefer outcome $g$ to outcome $\mathrm{b}$ at every state, i.e. $v^{1}(x)[\mathrm{b}]=v^{2}(x)[\mathrm{b}]=0$ and $v^{1}(x)[\mathrm{g}]=v^{1}(x)[\mathrm{g}]=1$ for all $x \in X$. For all $x=\left(x^{1}, x^{2}\right) \in X$ and $i=1,2$, the strategy chosen by $i$ at $x$ is $\sigma^{i}(x)=1$ if $x^{i}<0.5$ and $\sigma^{i}(x)=\mathrm{r}$ if $x^{i} \geq 0.5$. Finally, at every $x=\left(x^{1}, x^{2}\right) \in X$ and for $i=1,2$ and $j=3-i$, the belief $\beta^{i}(x) \in \Delta_{\mathrm{F}}(X)$ of player $i$ at $x$ is the product of the Dirac measure on $X^{i}$ centered at $x^{i}$ and the uniform distribution over $\left[0,2 x^{i}\right] \subseteq X^{j}$ if $x^{i}<0.5$ or over $\left[2 x^{i}-1,1\right]$ if $x^{i} \geq 0.5$. Thus, for instance, the belief of type $x^{1}=0.2$ of player 1 is the uniform distribution over $\{0.2\} \times[0,0.4]$, the belief of 
type $x^{2}=0.7$ of player 2 is the uniform distribution over $[0.4,1] \times\{0.7\}$, and so on. Now consider types $\widetilde{x}^{1}=0.3$ and $\widehat{x}^{1}=0.4$ of player 1 . Type $\widetilde{x}^{1}$ chooses strategy $\mid$ and believes $x^{2}$ is uniformly distributed over $[0,0.6]$; type $\hat{x}^{1}$ also chooses strategy $\mathrm{I}$, but believes $x^{2}$ is uniformly distributed over $[0,0.8]$. The two types have different first-order beliefs, since $\widetilde{x}^{1}$ attaches probability $5 / 6$ to player 2 choosing $u$, whereas $\hat{x}^{1}$ attaches probability $5 / 8$ to that event, nevertheless the two types have the same first-order preferences: they rank acts in $F(S)$ in the same way, since both strictly prefer $g$ to $b$, both are certain of their own strategy l, and both consider u more likely. Going to second-order preferences, however, the two types separate: $\widehat{x}^{1}$ attaches positive probability (namely, 1/16) to the event that $0.75<x^{2} \leq 0.8$, hence positive probability to the event that player 2 is certain that player 1 chooses strategy $r$, whereas $\widetilde{x}^{1}$ attaches probability zero to that event. Thus, for instance, $\widetilde{x}^{1}$ is indifferent (while $\widehat{x}^{1}$ is not) between the constant act $g$ and the act that gives $g$ if and only if player 2 is not indifferent between the constant act $g$ and the act that gives $g$ if and only if player 1 chooses $r$. Analogously, any two distinct elements of $X$ correspond to distinct elements of $\Omega_{\mathrm{SAV}}$ - as we indeed already knew from our theorems, since this type space is clearly non-redundant-although in order to prove this we might have to go even further up in the preference hierarchy. In other words, types having distinct $n$-order beliefs must have distinct $m$-order preferences for some $m \geq n$, although in general one does need some sufficiently large $m>n$. Note that in this example second- and higher-order beliefs are trivial (they are completely determined by first-order beliefs) and that all possible first-order beliefs are generated by the type space; more complicated examples would show that all possible $n$-order beliefs can be generated by some standard Savage type space.

\section{APPENDICES}

\section{A. Results ON SIMPLE SPACES}

When dealing with simple spaces in this appendix (and the ones to follow), we speak of open sets, continuous functions, and so on, without explicit reference to their topological structures; the understanding is that the relevant topology on the simple space under consideration is the one generated by the events in that space. Thus, the product of a finite family of simple spaces (resp. a subspace of a simple space) is understood to be endowed with the topology generated by the product algebra (resp. its relative algebra). No confusion can arise, as this is the same as the product topology (resp. the relative topology induced by the topology on the larger space).

LEMMA 1. A subset of a simple space is an event if and only if it is both closed and open. A subspace of a simple space is simple if and only if it is closed. ${ }^{28}$

Proof. An open set in a simple space is a union of events; thus, a closed (hence

\footnotetext{
${ }^{28} \mathrm{~A}$ topological space whose topology has a countable base of clopen sets is called zero-dimensional, and zero-dimensional compact Hausdorff topological spaces are called Boolean. Thus, what we call a simple space is a Boolean topological space stripped down to its algebra of clopen sets. For an excellent treatment of Boolean spaces, see Koppelberg (1989)—our proof of Lemma 1 is taken from there.
} 
compact) and open set in a simple space is the union of a finite family of events, hence an event. The other direction is obvious; each event and its complement are both open, hence both closed. Clearly, a subset of a simple space is compact Hausdorff if and only if it is closed in its relative topology. Since the latter is the same as the topology generated by its relative algebra, the second claim follows.

LEMmA 2. If $X$ is a simple space, then $\Pi(X)$ is a simple space.

Proof. Pick an injective function $v: F(X) \times F(X) \rightarrow \mathbb{N}$ such that $v^{-1}(n) \neq \varnothing$ for every $n \geq 1$ such that $v^{-1}(n+1) \neq \varnothing$. Such a function exists, as $X$ is simple and thus $F(X)$ is countable. Let $\bar{\Pi}(X)$ be the set of all subsets of $F(X) \times F(X)$. Define a metric on $\bar{\Pi}(X)$ by letting $d\left(\bar{\pi}, \bar{\pi}^{\prime}\right)=0$ if $\bar{\pi}=\bar{\pi}^{\prime}$ and $d\left(\bar{\pi}, \bar{\pi}^{\prime}\right)=1 / n$ if $n$ is the smallest $k \in \mathbb{N}$ such that $v^{-1}(k) \in \bar{\pi} \backslash \bar{\pi}^{\prime}$ or $v^{-1}(k) \in \bar{\pi}^{\prime} \backslash \bar{\pi}$. The induced topology makes $\bar{\Pi}(X)$ either discrete-this is if $X$ is actually finite, in which case $\bar{\Pi}(X)$ is also finite-or homeomorphic to $\{0,1\}^{\mathbb{N}}$. Thus, $\bar{\Pi}(X)$ is compact Hausdorff, and every $d$-open ball is also closed. For every $f, g \in$ $F(X)$ let

$$
\bar{\Pi}_{f, g}(X)=\{\bar{\pi} \in \bar{\Pi}(X):(f, g) \in \bar{\pi}\} .
$$

If $v(f, g)=n$ and $P_{n}$ is the set of all maps $p:\{1, \ldots, n\} \rightarrow\{0,1\}$ with $p(n)=1$, then

$$
\bar{\Pi}_{f, g}(X)=\bigcup_{p \in P_{n}}\left\{\bar{\pi} \in \bar{\Pi}(X): v^{-1}\left(p^{-1}(1)\right) \subseteq \bar{\pi} \text { and } v^{-1}\left(p^{-1}(0)\right) \cap \bar{\pi}=\varnothing\right\} .
$$

This shows $\bar{\Pi}_{f, g}(X)$ is a finite union of $d$-open balls, hence both closed and open. Conversely, every $d$-open ball can be written, for some $\bar{\pi} \in \bar{\Pi}(X)$ and some $n$, as

$$
\bigcap_{k=1}^{n}\left\{\bar{\pi}^{\prime} \in \bar{\Pi}(X):\left\{v^{-1}(k)\right\} \cap \bar{\pi}^{\prime}=\left\{v^{-1}(k)\right\} \cap \bar{\pi}\right\} .
$$

Thus, every $d$-open ball is an event in the algebra on $\bar{\Pi}(X)$ generated by the sets $\bar{\Pi}_{f, g}(X)$. We conclude that $\bar{\Pi}(X)$ equipped with this algebra is simple. It remains to prove $\Pi(X)$ is $d$-closed. This is immediate, as $\Pi(X)$ is an intersection of closed subsets of $\bar{\Pi}(X)$, i.e. the intersection of all sets having either the form $\bar{\Pi}_{f, f}(X)$ where $f \in F(X)$-guaranteeing reflexivity —or the form

$$
\bar{\Pi}_{f, h}(X) \cup\left(\bar{\Pi}(X) \backslash \bar{\Pi}_{f, g}(X)\right) \cup\left(\bar{\Pi}(X) \backslash \bar{\Pi}_{g, h}(X)\right)
$$

where $f, g, h \in F(X)$-this guarantees transitivity.

Lemma 3. Let $X$ and $Y$ be simple spaces and let $\varphi: X \rightarrow Y$. Then $\varphi$ is measurable if and only if it is continuous. In this case the induced $\widehat{\varphi}: \Pi(X) \rightarrow \Pi(Y)$ is also continuous.

Proof. The second statement follows at once from the first and from Lemma 2. Sufficiency in the first statement is obvious, so let us assume $\varphi$ is measurable and prove necessity. Pick any open $A \subseteq Y$. Since $Y$ is simple, there are clopen subsets $A_{n}$ of $Y$ such that $A=\cup_{n} A_{n}$. By Lemma 1, each $A_{n}$ is an event, so each $\varphi^{-1}\left(A_{n}\right)$ is an event and hence (again by that lemma) both closed and open. This implies $\varphi^{-1}(A)=\cup_{n} \varphi^{-1}\left(A_{n}\right)$ is open. 
Lemma 4. Let $X$ and $Y$ be uncertainty spaces. Assume $X$ is simple and let $\varphi: X \rightarrow Y$ be a measurable function. If $\varphi$ is onto, then the induced mapping $\widehat{\varphi}: \Pi(X) \rightarrow \Pi(Y)$ is onto.

Proof. Assume that $\varphi: X \rightarrow Y$ is onto, and let $\pi \in \Pi(Y)$. We have to find a preference relation on $F(X)$ that is mapped into $\pi$ by the function $\widehat{\varphi}$. Consider the set

$$
\{(f \circ \varphi, g \circ \varphi):(f, g) \in \pi\} \cup\{(f, f): f \in F(X)\} .
$$

This is a well-defined binary relation on $F(X)$, as ontoness of $\varphi$ ensures that, if $f, f^{\prime} \in$ $F(Y)$ and $f \circ \varphi=f^{\prime} \circ \varphi$, then $f=f^{\prime}$. Since it contains $(f, f)$ for every $f \in F(X)$, it satisfies reflexivity, whereas transitivity is directly inherited from $\pi$.

\section{B. Proofs for Section 3}

Lemma 5. Let $n \geq 0$. The projection $\rho_{n}: \Omega_{n+1} \rightarrow \Omega_{n}$ is onto. For all $\omega_{n} \in \Omega_{n}$ the set $\rho_{n}^{-1}\left(\omega_{n}\right)$ has, in fact, at least two distinct elements. (In particular, $\Omega$ has the cardinality of the continuum.) The induced $\widehat{\rho}_{n}: \Pi\left(\Omega_{n+1}\right) \rightarrow \Pi\left(\Omega_{n}\right)$ is onto.

Proof. The third statement follows from the first, using Lemma 4 and the fact that $\Omega_{n+1}$ is finite, hence simple. The second statement implies the first and, since $|Z| \geq 2$, it is obviously true for $n=0$. Proceeding inductively, let $n \geq 0$ and suppose that, for all $\omega_{n} \in \Omega_{n}$, the set $\rho_{n}^{-1}\left(\omega_{n}\right)$ has at least two distinct elements. Fix $\omega_{n+1} \in \Omega_{n+1}$ and consider, for each player $i$, the preference relations

$$
\begin{aligned}
& \widehat{\pi}^{i}=\left\{(f, f): f \in F\left(\Omega_{n+1}\right)\right\} \cup\left\{\left(f \circ \rho_{n}, g \circ \rho_{n}\right):(f, g) \in \delta_{n+1}^{i}\left(\omega_{n+1}\right)\right\} \\
& \tilde{\pi}^{i}=\widehat{\pi}^{i} \cup\left\{\left(f^{\prime}, g^{\prime}\right)\right\}
\end{aligned}
$$

where $f^{\prime}$ and $g^{\prime}$ are arbitrarily chosen, distinct acts in $F\left(\Omega_{n+1}\right)$ such that $f^{\prime} \neq f \circ \rho_{n} \neq g^{\prime}$ for all $f \in F\left(\Omega_{n}\right)$. (Such $f^{\prime}$ and $g^{\prime}$ exist due to the induction hypothesis.) These are distinct preference relations, and clearly $\widehat{\rho}_{n}\left(\widehat{\pi}^{i}\right)=\widehat{\rho}_{n}\left(\widetilde{\pi}^{i}\right)=\delta_{n+1}^{i}\left(\omega_{n+1}\right)$. Thus, $\left(\omega_{n+1},\left(\widehat{\pi}^{i}\right)_{i \in I}\right)$ and $\left(\omega_{n+1},\left(\tilde{\pi}^{i}\right)_{i \in I}\right)$ are distinct elements of $\Omega_{n+2}$.

LEMma 6. $(\Omega, \mathscr{A})$ is a simple uncertainty space.

Proof. The sequence $\left(\Omega_{n}, \rho_{n}\right)$ is an inverse (or projective) system of finite (hence compact Hausdorff) topological spaces (see e.g. Bourbaki 1989, p. 48). By construction, the set $\Omega$ endowed with the relative product topology, which has $\mathscr{A}$ as a base, is the inverse (or projective) limit of the system, hence compact Hausdorff (see e.g. Bourbaki 1989, p. 78).

Proof of Proposition 1. As a preliminary step, we prove that for every $f \in F(\Omega)$ there exist $n \geq 0$ and $g \in F\left(\Omega_{n}\right)$ such that $f=g \circ \varrho_{n}$. Indeed, by definition of $\mathscr{A}$, for every $z \in Z$ there exist $n_{z} \geq 0$ and $E_{z} \subseteq \Omega_{n_{z}}$ such that $f^{-1}(z)=\varrho_{n_{z}}^{-1}\left(E_{z}\right)$. Letting $n=\max _{z \in Z} n_{z}$ we see that the sets of the form $\rho_{n}^{-1}\left(\cdots\left(\rho_{n_{z}}^{-1}\left(E_{z}\right)\right)\right)$, where $z \in Z$, partition $\Omega_{n}$. Thus, $f=$ $g \circ \varrho_{n}$, where $g \in F\left(\Omega_{n}\right)$ maps each $\omega_{n} \in \Omega_{n}$ into the unique $z \in Z$ whose corresponding element of the partition contains $\omega_{n}$. The proof of the preliminary step is complete. 
By our definitions, for each player $i$ and all $n \geq 1$ we have $\delta_{n}^{i} \circ \varrho_{n}=\widehat{\rho}_{n-1} \circ \delta_{n+1}^{i} \circ \varrho_{n+1}$. Furthermore, by induction using the latter, for all $m \geq n \geq 1$ we have

$$
\delta_{n}^{i} \circ \varrho_{n}=\widehat{\rho}_{n-1} \circ \cdots \circ \widehat{\rho}_{m-1} \circ \delta_{m+1}^{i} \circ \varrho_{m+1} .
$$

Define the mapping $\varpi: \Omega \rightarrow \Pi^{I}(\Omega)$ as follows: for every player $i$ and every $\omega \in \Omega$,

$$
\varpi^{i}(\omega)=\left\{\left(f \circ \varrho_{n}, g \circ \varrho_{n}\right): n \geq 0,(f, g) \in \delta_{n+1}^{i}\left(\varrho_{n+1}(\omega)\right)\right\} .
$$

We must prove the latter is well defined and belongs to $\Pi(\Omega)$. To verify it is well defined, note that for every $m \geq n \geq 0$, every $f, g \in F\left(\Omega_{n}\right)$, and every $f^{\prime}, g^{\prime} \in F\left(\Omega_{m}\right)$ such that $f \circ \varrho_{n}=f^{\prime} \circ \varrho_{m}$ and $g \circ \varrho_{n}=g^{\prime} \circ \varrho_{m}$, one has $f^{\prime}=\rho_{n} \circ \cdots \circ \rho_{m-1} \circ f$ and $g^{\prime}=\rho_{n} \circ \cdots \circ \rho_{m-1} \circ g$ (these follow from ontoness of $\varrho_{m}$ and from $\varrho_{n}=\rho_{n} \circ \cdots \circ \rho_{m-1} \circ \varrho_{m}$ ). Hence, by (7), one has $(f, g) \in \delta_{n+1}^{i}\left(\varrho_{n+1}(\omega)\right)$ if and only if $\left(f^{\prime}, g^{\prime}\right) \in \delta_{m+1}^{i}\left(\varrho_{m+1}(\omega)\right)$. To verify it belongs to $\Pi(\Omega)$, just note that reflexivity and transitivity are immediate consequences of the preliminary step and of the corresponding properties of $\delta_{n+1}^{i}\left(\varrho_{n+1}(\omega)\right)$ for all $n \geq 0$. The preliminary step also guarantees that for any two distinct preference relations in $\Pi(\Omega)$ there exist $n \geq 0$ and $f, g \in F\left(\Omega_{n}\right)$ such that $\left(f \circ \varrho_{n}, g \circ \varrho_{n}\right)$ is an element of one relation but not of the other. Thus the property that $\widehat{\varrho}_{n-1} \circ \varpi^{i}=\delta_{n}^{i} \circ \varrho_{n}$ for all $n \geq 1$, which $\varpi^{i}$ satisfies by definition, uniquely identifies $\varpi^{i}$. Moreover, by definition of $\varpi^{i}$, for all $n \geq 1$ and all $f, g \in F\left(\Omega_{n-1}\right)$ one has

$$
\left\{\omega \in \Omega:\left(f \circ \varrho_{n-1}, g \circ \varrho_{n-1}\right) \in \varpi^{i}(\omega)\right\}=\left\{\omega \in \Omega:(f, g) \in \delta_{n}^{i}\left(\varrho_{n}(\omega)\right)\right\} .
$$

The latter proves (again by the preliminary step) that $\varpi$ is measurable.

Proof of Proposition 2. Let $\gamma$ be the generator of $(X, \mathscr{B}, \sigma, \vartheta)$. Let $\mathscr{B}^{\prime}=\left\{\gamma^{-1}(A): A \in\right.$ $\mathscr{A}$ \}. We must prove that $\mathscr{B}^{\prime}$ is the smallest algebra of subsets of $X$ satisfying (1) and (2). Now (1) is obvious, since $\sigma=\varrho_{0} \circ \gamma$. To prove (2), let $\widehat{\gamma}$ denote the mapping from $\Pi(X, \mathscr{B})$ to $\Pi(\Omega, \mathscr{A})$ induced by $\gamma$. By (3), for all $f, g \in F(\Omega, \mathscr{A})$ we have

$$
\left\{x \in X:(f \circ \gamma, g \circ \gamma) \in \vartheta^{i}(x)\right\}=\gamma^{-1}\left(\left\{\omega \in \Omega:(f, g) \in \varpi^{i}(\omega)\right\}\right) .
$$

This proves (2), as by definition of $\mathscr{B}^{\prime}$ every act in $F\left(X, \mathscr{B}^{\prime}\right)$ can be written as $f \circ \gamma$ for some $f \in F(\Omega, \mathscr{A})$. If $\mathscr{B}^{\prime \prime}$ is another algebra satisfying (1) and (2), then $\gamma^{-1}\left(\varrho_{n}^{-1}(A)\right) \in \mathscr{B}^{\prime \prime}$ for all $n \geq 0$ and all $A \subseteq \Omega_{n}$. For $n=0$ this follows at once from (1), hence by induction using (2) it is true for $n>0$ as well. Thus, $\mathscr{B}^{\prime} \subseteq \mathscr{B}^{\prime \prime}$. This proves the first claim. To prove the second claim, just observe that, by the first claim in this proposition and the definition of $\mathscr{A}$, non-redundancy holds if and only if for all distinct $x, x^{\prime} \in X$ there exists $n \geq 0$ such that $\varrho_{n}(\gamma(x)) \neq \varrho_{n}\left(\gamma\left(x^{\prime}\right)\right)$, and such $n$ exists if and only if $\gamma(x) \neq \gamma\left(x^{\prime}\right)$.

Proof of Theorem 1. By the preliminary step in the proof of Proposition 1, it is enough to show that the mapping from $\Omega$ to $S \times \Pi^{I}(\Omega)$ such that $\omega \mapsto\left(\varrho_{0}(\omega), \varpi(\omega)\right)$ is a bijection. Choose any $s \in S$ and any $\left(\pi^{i}\right)_{i \in I} \in \Pi^{I}(\Omega)$, and define $\omega=\left(\omega_{0}, \omega_{1}, \ldots\right) \in \Omega$ by letting $\omega_{0}=$ $s$ and recursively defining $\omega_{n+1}=\left(\omega_{n},\left(\varrho_{n}\left(\pi^{i}\right)_{i \in I}\right)\right)$ for all $n \geq 0$. Clearly, $\varpi^{i}(\omega)=\pi^{i}$ for all $i \in I$, thus ontoness is established. To prove injectivity, note that for every two distinct 
$\omega, \omega^{\prime} \in \Omega$ there exists $n \geq 0$ such that $\varrho_{n}(\omega) \neq \varrho_{n}\left(\omega^{\prime}\right)$, hence either $\varrho_{0}(\omega) \neq \varrho_{0}\left(\omega^{\prime}\right)$ or there exist some $n \geq 1$ and some player $i$ such that $\delta_{n}^{i}\left(\varrho_{n}(\omega)\right) \neq \delta_{n}^{i}\left(\varrho_{n}\left(\omega^{\prime}\right)\right)$, and in the latter case $\varpi(\omega) \neq \varpi\left(\omega^{\prime}\right)$ by the preliminary step in the proof of Proposition 1 .

Proof of Proposition 3. Let $\iota: \Omega^{\prime} \rightarrow \Omega$ denote inclusion. Note that (iii) is equivalent to

$$
\{(f, g) \in F(\Omega) \times F(\Omega): f \circ \iota=g \circ \iota\} \subseteq \varpi^{i}(\iota(\omega)) \quad \forall i \in I, \forall \omega \in \Omega^{\prime} .
$$

It is clear that if $\Omega^{\prime}$ satisfies (i) then it must satisfy (ii) as well. Now suppose $\Omega^{\prime}$ satisfies (ii), i.e. it is generated by some structure $\mathscr{X}=(X, \mathscr{B}, \sigma, \vartheta)$, and let $\gamma$ be the generator of $\mathscr{X}$, so that $\Omega^{\prime}=\gamma(X)$. Then for all $x \in X$, by reflexivity of $\vartheta^{i}(x)$, we have $(f \circ \gamma, g \circ \gamma) \in \vartheta^{i}(x)$ for all $i \in I$ and all $f, g \in F(\Omega)$ that coincide on $\Omega^{\prime}$. This means that $\Omega^{\prime}$ satisfies (8) and hence (iii). Finally, assume $\Omega^{\prime}$ satisfies (iii), or equivalently (8), and let $\mathscr{A}^{\prime}=\left\{A \cap \Omega^{\prime}: A \in \mathscr{A}\right\}$. Then the mapping $\varpi^{\prime}: \Omega^{\prime} \rightarrow \Pi^{I}\left(\Omega^{\prime}, \mathscr{A}^{\prime}\right)$ such that

$$
\omega \mapsto\left(\left\{(f \circ \iota, g \circ \iota) \in F\left(\Omega^{\prime}\right) \times F\left(\Omega^{\prime}\right):(f, g) \in \varpi^{i}(\iota(\omega))\right\}\right)_{i \in I}
$$

is well defined and, together with the mapping $\varrho_{0} \circ \iota$, it makes $\Omega^{\prime}$ into the canonical substructure $\left(\Omega^{\prime}, \mathscr{A}^{\prime}, \varrho_{0}^{\prime}, \varpi^{\prime}\right)$, which proves that (i) holds.

Proof of Proposition 4. Sufficiency is obvious. To prove necessity, let $\mathscr{X}=(X, \mathscr{B}, \sigma, \vartheta)$ be a minimal and non-redundant structure, let $\Omega^{\prime}$ be its generated set of hierarchies, and let $\gamma$ be its generator. Then $\gamma$ is injective by non-redundancy and Proposition 2, so the mapping $\eta: X \rightarrow \Omega^{\prime}$ such that $x \mapsto \gamma(x)$ is a bijection. By minimality, and again by Proposition 2, the inverse of $\eta$ is measurable. Thus, the induced mapping $\widehat{\eta}: \Pi(X) \rightarrow \Pi\left(\Omega^{\prime}\right)$ is also measurable and has a measurable inverse-namely, the mapping from $\Pi\left(\Omega^{\prime}\right)$ to $\Pi(X)$ induced by $\eta^{-1}$. Therefore, it suffices to show that $\eta$ is a morphism from $\mathscr{X}$ to the canonical substructure $\left(\Omega^{\prime}, \mathscr{A}^{\prime}, \varrho_{0}^{\prime}, \varpi^{\prime}\right)$ induced by $\Omega^{\prime}$, i.e. we must prove that

$$
\widehat{\eta} \circ \vartheta^{i}=\varpi^{i} \circ \eta
$$

for every player $i$. Let $\iota: \Omega^{\prime} \rightarrow \Omega$ denote inclusion. Since $\left(\Omega^{\prime}, \mathscr{A}^{\prime}\right)$ is a subspace of $(\Omega, \mathscr{A})$, every $f \in F\left(\Omega^{\prime}, \mathscr{A}^{\prime}\right)$ can be written as $f=g \circ \iota$ for some $g \in F(\Omega, \mathscr{A})$. This implies that the mapping $\widehat{\imath}: \Pi\left(\Omega^{\prime}, \mathscr{A}^{\prime}\right) \rightarrow \Pi(\Omega, \mathscr{A})$ induced by $\iota$ is injective, so in order to prove (9) it suffices to prove that $\widehat{\imath} \circ \widehat{\eta} \circ \vartheta^{i}=\widehat{\imath} \circ \varpi^{\prime i} \circ \eta$. Indeed,

$$
\begin{aligned}
& \widehat{\imath} \circ \widehat{\eta} \circ \vartheta^{i}=\widehat{\gamma} \circ \vartheta^{i} \quad \text { (because } \gamma=\iota \circ \eta \text { ) } \\
& =\varpi^{i} \circ \gamma \quad \text { (because } \gamma \text { is a morphism) } \\
& =\varpi^{i} \circ \iota \circ \eta \quad \text { (again because } \gamma=\iota \circ \eta \text { ) } \\
& =\widehat{\iota} \circ \varpi^{\prime i} \circ \eta \text {. }
\end{aligned}
$$

(The last equality follows from the fact that $\Omega^{\prime}$ induces a canonical substructure and, therefore, the inclusion mapping $\iota$ is a morphism.)

\section{Proofs for Section 4}

The result in Proposition 5 relies only on the fact that $(\Omega, \mathscr{A})$ is a simple space. Indeed, it is a special case of Lemma 8 below, which in turn relies on the following result. 
LEMma 7. Let $X$ be a space and let $\pi \in \Pi(X)$ be monotone continuous. Take a sequence of events $A_{n}$ such that $A=\cup_{n} A_{n}$ is also an event. Then $A$ is $\pi$-null if and only if each $A_{n}$ is $\pi-$ null.

Proof. Necessity is obvious. For sufficiency, suppose each $A_{n}$ is $\pi$-null but $A$ is not. Then there exist $f, g, h \in F(X)$ such that

$$
(f A h, g A h) \dot{\epsilon} \pi
$$

Let $B_{n}=A_{1} \cup \cdots \cup A_{n}$ for all $n \geq 1$. Identify $Z$ with the set $\{1, \ldots,|Z|\}$ and let $K=\max \{z \in$ $\left.Z: g^{-1}(z) \neq \varnothing\right\}$. For all $1 \leq k \leq K$ and all $n \geq 1$, let

$$
C_{k}=\{x \in X: 1 \leq j \leq k, g(x)=j\} \quad \text { and } \quad D_{k}^{n}=C_{k} \cap\left(A \backslash B_{n}\right)
$$

and write $g_{k}^{n}$ for the act that coincides with $g$ on $D_{k}^{n}$ and with $f A h$ everywhere else. Note that $g_{K}^{n}$ and $g A h$ only differ on the $\pi$-null event $B_{n}$, so

$$
\left(g A h, g_{K}^{n}\right) \in \pi \quad \forall n \geq 1 .
$$

As $D_{1}^{n} \downarrow \varnothing$, by (10) and monotone continuity of $\pi$ we have $\left(g_{1}^{n_{1}}, g A h\right) \dot{\epsilon} \pi$ for some $n_{1} \geq 1$. Inductively, let $1 \leq k<K$ and assume we found $n_{k} \geq 1$ such that $\left(g_{k}^{n_{k}}, g A h\right) \dot{\epsilon} \pi$. Since $D_{k+1}^{n} \downarrow \varnothing$, by monotone continuity of $\pi$ there is $n_{k+1}>n_{k}$ such that

$$
\left(g_{k+1}^{n_{k+1}} D_{k+1}^{n_{k+1}} g_{k}^{n_{k}}, g A h\right) \dot{\epsilon} \pi .
$$

But $g_{k+1}^{n_{k+1}} D_{k+1}^{n_{k+1}} g_{k}^{n_{k}}$ and $g_{k+1}^{n_{k+1}}$ only differ on the $\pi$-null event $D_{k}^{n_{k}} \backslash D_{k+1}^{n_{k+1}}$, so

$$
\left(g_{k+1}^{n_{k+1}}, g_{k+1}^{n_{k+1}} D_{k+1}^{n_{k+1}} g_{k}^{n_{k}}\right) \in \pi .
$$

By (12), (13), and transitivity of $\pi,\left(g_{k+1}^{n_{k+1}}, g A h\right) \dot{\epsilon} \pi$, contradicting (11) for $k=K-1$.

LeMma 8. Let $(X, \mathscr{B})$ be a simple space. Then every $\pi \in \Pi(X, \mathscr{B})$ is monotone continuous. Let $\pi \in \Pi(X, \mathscr{B})$ and let $A_{1}, A_{2}, \ldots$ be $\pi$-null events in $\mathscr{B}$. Let $A=\cup_{n} A_{n}$ and let $\mathscr{B}^{+}$be the algebra generated by $\mathscr{B} \cup\{A\}$. There is a unique monotone continuous $\pi^{+} \in \Pi\left(X, \mathscr{B}^{+}\right)$ such that

$$
\pi^{+} \cap[F(X, \mathscr{B}) \times F(X, \mathscr{B})]=\pi .
$$

Moreover, if the union $A^{\prime}$ of any sequence of events $A_{1}^{\prime}, A_{2}^{\prime}, \ldots$ in $\mathscr{B}$ satisfies $A^{\prime} \in \mathscr{B}{ }^{+}$, then $A^{\prime}$ is $\pi^{+}$-null if and only if $A_{1}^{\prime}, A_{2}^{\prime}, \ldots$ are all $\pi$-null. In particular, $A$ is $\pi^{+}$-null.

Proof. For the first statement, just note that every sequence of events $E_{n}$ in $\mathscr{B}$ such that $E_{n} \downarrow \varnothing$ must satisfy $E_{n}=\varnothing$ for every $n$ sufficiently large. Indeed, by definition of a simple space, in the topology on $X$ generated by $\mathscr{B}, X$ is compact, each $E_{n}$ is closed, and the sequence $E_{n}$ has the finite intersection property. The proof of the second part has four steps. In the first step we construct the binary relation $\pi^{+}$and prove that it satisfies (14). In the second step we prove that $\pi^{+}$is a monotone continuous preference relation. 
In the third step we prove the last part of the proposition. In the fourth step we prove uniqueness.

Step 1. Every element of $\mathscr{B}^{+}$has the form $(B \cap A) \cup(C \backslash A)$ where $B, C \in \mathscr{B}$. The latter is immediate, since $\mathscr{B}^{+}$must clearly include the family of sets of such form, and this family is easily seen to be an algebra. Thus, for each $f_{+} \in F\left(X, \mathscr{B}^{+}\right)$we can pick two acts $\psi_{f_{+}}$and $\phi_{f_{+}}$in $F(X, \mathscr{B})$ such that $\psi_{f_{+}}$coincides with $f_{+}$on $X \backslash A$ and $\phi_{f_{+}}$coincides with $f_{+}$on $A$. Now let

$$
\pi^{+}=\left\{\left(f_{+}, g_{+}\right) \in F\left(X, \mathscr{B}^{+}\right) \times F\left(X, \mathscr{B}^{+}\right):\left(\psi_{f_{+}}, \psi_{g_{+}}\right) \in \pi\right\} .
$$

To prove (14), first note that if $f, g \in F(X, \mathscr{B})$ coincide on $X \backslash A$, then the event

$$
A_{f, g}=\{x \in X: f(x) \neq g(x)\}
$$

satisfies $A_{f, g} \subseteq\left(A_{1} \cup \cdots \cup A_{n}\right)$ for some $n$ and is thus null according to $\pi$. Indeed, suppose to the contrary that $A_{f, g} \nsubseteq\left(A_{1} \cup \cdots \cup A_{n}\right)$ for every $n$. Then $A_{f, g} \backslash\left(A_{1} \cup \cdots \cup A_{n}\right)$ is a strictly decreasing sequence of events. Since $X$ is simple, this sequence has nonempty intersection, contradicting the assumption that $f$ and $g$ coincide on $X \backslash A$, since the latter implies $A_{f, g} \subseteq A$. We have thus shown that for all $f, g \in F(X, \mathscr{B})$ that coincide on $X \backslash A$ we have $(f, g) \in \pi$. This has two implications. The first implication is that $A$ is null according to $\pi^{+}$. Indeed, for all $f_{+}, g_{+} \in F\left(X, \mathscr{B}^{+}\right)$that coincide on $X \backslash A$ we must have $\left(\psi_{f_{+}}, \psi_{g_{+}}\right) \in \pi$ and hence also $\left(f_{+}, g_{+}\right) \in \pi^{+}$. The second implication is that for all $f, g \in F(X, \mathscr{B})$ we have $\left(f, \psi_{f}\right) \in \pi \ni\left(\psi_{f}, f\right)$ and $\left(g, \psi_{g}\right) \in \pi \ni\left(\psi_{g}, g\right)$, hence, by transitivity of $\pi,(f, g) \in \pi$ if and only if $(f, g) \in \pi^{+}$. This proves (14).

Step 2. Reflexivity and transitivity of $\pi^{+}$follow directly from the corresponding properties of $\pi$. To prove monotone continuity, let $z \in Z$, let $B_{n}$ and $C_{n}$ be sequences in $\mathscr{B}$ such that the sequence $D_{n}=\left(B_{n} \cap A\right) \cup\left(C_{n} \backslash A\right)$ has empty intersection, and choose $f_{+}, g_{+} \in F\left(X, \mathscr{B}^{+}\right)$such that $\left(f_{+}, g_{+}\right) \dot{\epsilon} \pi^{+}$. Then $\left(\psi_{f_{+}}, \psi_{g_{+}}\right) \dot{\epsilon} \pi$, and since the sequence $E_{n}=C_{n} \backslash\left(A_{1} \cup \cdots \cup A_{n}\right)$ also has an empty intersection, by monotone continuity of $\pi$ we have $\left(z E_{n} \psi_{f_{+}}, \psi_{g_{+}}\right) \dot{\epsilon} \pi$ and $\left(\psi_{f_{+}}, z E_{n} \psi_{g_{+}}\right) \dot{\epsilon} \pi$ for all $n$ sufficiently large. The two acts in each of the pairs $\left(z D_{n} f_{+}, z E_{n} \psi_{f_{+}}\right),\left(z E_{n} \psi_{g_{+}}, z D_{n} g_{+}\right),\left(\psi_{g_{+}}, g_{+}\right)$, and $\left(f_{+}, \psi_{f_{+}}\right)$coincide on $X \backslash A$. Since $A$ is null according to $\pi^{+}$, by transitivity of $\pi^{+}$we obtain $\left(z D_{n} f_{+}, g_{+}\right) \dot{\epsilon} \pi^{+}$ and $\left(f_{+}, z D_{n} g_{+}\right) \dot{\epsilon} \pi^{+}$for all $n$ sufficiently large, thus establishing monotone continuity of $\pi^{+}$.

Step 3. Here we prove that a union of events in $\mathscr{B}$ that belongs to $\mathscr{B}^{+}$is $\pi^{+}$-null if and only if each event in the union is $\pi$-null. Since $\pi^{+}$satisfies (14), necessity is obvious. In order to prove sufficiency, by Lemma 7 it suffices to show that if $E \in \mathscr{B}$ is $\pi$-null then $E$ is also $\pi^{+}$-null. Thus, let $f_{+}, g_{+}, h_{+} \in F\left(X, \mathscr{B}^{+}\right)$. Since $E \cap A$ is $\pi^{+}$-null (because so is $A$, by Step 1) and $f_{+}$and $\psi_{f_{+}}$coincide on $E \backslash A$, and since $A$ is $\pi^{+}$-null and $h_{+}$and $\psi_{h_{+}}$ coincide on $X \backslash A$, we have $\left(f_{+} E h_{+}, \psi_{f_{+}} E \psi_{h_{+}}\right) \in \pi^{+}$. Similarly, $\left(\psi_{g_{+}} E \psi_{h_{+}}, g_{+} E h_{+}\right) \in \pi^{+}$. But $E$ is $\pi$-null, so $\left(\psi_{f_{+}} E \psi_{h_{+}}, \psi_{g_{+}} E \psi_{h_{+}}\right) \in \pi$, hence $\left(\psi_{f_{+}} E \psi_{h_{+}}, \psi_{g_{+}} E \psi_{h_{+}}\right) \in \pi^{+}$by (14), so $\left(f_{+} E h_{+}, g_{+} E h_{+}\right) \in \pi^{+}$by transitivity of $\pi^{+}$. This shows that every event in $\mathscr{B}$ that is null according to $\pi$ is also null according to $\pi^{+}$.

Step 4. In order to prove uniqueness, by the first part of the proof it is clearly enough to show the following: $A$ is null according to every monotone continuous preference 
relation $\pi^{\prime}$ on $F\left(X, \mathscr{B}^{+}\right)$that, for all $f, g \in F(X, \mathscr{B})$, satisfies $(f, g) \in \pi^{\prime}$ if and only if $(f, g) \in \pi$. Suppose by contradiction that $\pi^{\prime}$ satisfies the latter but $A$ is not $\pi^{\prime}$-null. Then there are $f_{+}, g_{+}, h_{+} \in F\left(X, \mathscr{B}^{+}\right)$such that $\left(f_{+} A h_{+}, g_{+} A h_{+}\right) \dot{\epsilon} \pi^{\prime}$, hence $f, g, h \in F(X, \mathscr{B})$ such that

$$
(f A h, g A h) \dot{\epsilon} \pi^{\prime} .
$$

(For instance, let $h=\psi_{h_{+}}, f=\phi_{f_{+}}$, and $g=\phi_{g_{+}}$.) Now let $A_{n}^{\prime}=A_{1} \cup \cdots \cup A_{n}$ for all $n \geq 1$, define $C_{k}^{\prime}=h^{-1}(k)$ and $D_{k}^{n}=C_{k}^{\prime} \cap\left(A \backslash A_{n}^{\prime}\right)$ for all $k \in Z$ and all $n \in \mathbb{N}$, identify $Z$ with the set $\{1, \ldots,|Z|\}$, and let $K=\max \left\{k \in Z: C_{k}^{\prime} \neq \varnothing\right\}$. Since $D_{1}^{n} \downarrow \varnothing$ as $n \rightarrow \infty$ and $h$ is constant on $D_{1}^{n}$ for all $n$, by (15) and monotone continuity of $\pi^{\prime}$ there exists $n_{1} \geq 1$ such that $\left(f\left(A \backslash D_{1}^{n_{1}}\right) h, g A h\right) \dot{\epsilon} \pi^{\prime}$. Proceeding inductively for all $1 \leq k<K$, assume that we have found numbers $n_{k}>\cdots>n_{1} \geq 1$ such that, letting $E_{k}^{\prime}=A \backslash\left(D_{1}^{n_{1}} \cup \cdots \cup D_{k}^{n_{k}}\right)$, we have $\left(f E_{k}^{\prime} h, g A h\right) \dot{\epsilon} \pi^{\prime}$. Since $D_{k+1}^{n} \downarrow \varnothing$ as $n \rightarrow \infty$ and $h$ is constant on $D_{k+1}^{n}$ for all $n$, again by monotone continuity of $\pi^{\prime}$ there exists $n_{k+1}>n_{k}$ such that, letting $E_{k+1}^{\prime}=$ $E_{k}^{\prime} \backslash D_{k+1}^{n_{k+1}}$, we have $\left(f E_{k+1}^{\prime} h, g A h\right) \dot{\epsilon} \pi^{\prime}$. Now perform another induction as follows. Again because $D_{1}^{n} \downarrow \varnothing$ as $n \rightarrow \infty$ and $h$ is constant on $D_{1}^{n}$ for all $n$, by monotone continuity there exists $m_{1} \geq 1$ such that $\left(f E_{K}^{\prime} h, g\left(A \backslash D_{1}^{m_{1}}\right) h\right) \dot{\in} \pi^{\prime}$. Proceeding inductively for all $1 \leq k<K$, assume that we have found numbers $m_{k}>\cdots>m_{1} \geq 1$ such that, letting $E_{k}^{\prime \prime}=A \backslash\left(D_{1}^{m_{1}} \cup \cdots \cup D_{k}^{m_{k}}\right)$, we have $\left(f E_{K}^{\prime} h, g E_{k}^{\prime \prime} h\right) \dot{\epsilon} \pi^{\prime}$. Again because $D_{k+1}^{n} \downarrow \varnothing$ as $n \rightarrow \infty$ and $h$ is constant on $D_{k+1}^{n}$ for all $n$, by monotone continuity there exists $m_{k+1}>m_{k}$ such that, letting $E_{k+1}^{\prime \prime}=E_{k}^{\prime \prime} \backslash D_{k+1}^{m_{k+1}}$, we have $\left(f E_{K}^{\prime} h, g E_{k+1}^{\prime \prime} h\right) \dot{\epsilon} \pi^{\prime}$. But, since both $E_{K}^{\prime}=$ $\cup_{1 \leq k \leq K}\left(C_{k}^{\prime} \cap A_{n_{k}}^{\prime}\right)$ and $E_{K}^{\prime \prime}=\cup_{1 \leq k \leq K}\left(C_{k}^{\prime} \cap A_{m_{k}}^{\prime}\right)$ are events in $\mathscr{B}$, the acts $f E_{K}^{\prime} h$ and $g E_{K}^{\prime \prime} h$ both belong to $F(X, \mathscr{B})$. We conclude that $\left(f E_{K}^{\prime} h, g E_{K}^{\prime \prime} h\right) \dot{\in} \pi$. Since $E_{K}^{\prime} \subseteq A$ and $E_{K}^{\prime \prime} \subseteq A$, this contradicts our earlier conclusion that $\pi$ contains all pairs of acts coinciding on $X \backslash A$.

Proof of Proposition 5. As $(\Omega, \mathscr{A})$ is simple, the result is a special case of Lemma 8.

Lemma 9. Let $E, E^{\prime}, E_{1}, E_{2}, \ldots$ be closed subsets of $\Omega$. Then

(i) $M C_{m}\left(\cap_{n} E_{n}\right)=\cap_{n} M C_{m}\left(E_{n}\right)$ for every $m \geq 1$.

(ii) If $E \subseteq E^{\prime}$, then $M C_{1}(E) \subseteq M C_{1}\left(E^{\prime}\right)$.

(iii) If $E \subseteq M C_{1}(E)$, then $E \subseteq C C(E)$.

Proof. For every $n \geq 1$ take a sequence of events $A_{n, k}$ in $\mathscr{A}$ such that $E_{n}=\cap_{k} A_{n, k}$. Part (i) holds for $m=1$, since by definition of 1-mutual certainty we have $M C_{1}\left(\cap_{n} E_{n}\right)=$ $M C_{1}\left(\cap_{n} \cap_{k} A_{n, k}\right)=\cap_{n} \cap_{k} M C_{1}\left(A_{n, k}\right)=\cap_{n} M C_{1}\left(\cap_{k} A_{n, k}\right)=\cap_{n} M C_{m}\left(E_{n}\right)$. Now suppose part (i) holds up to some $m \geq 1$. Then $M C_{m+1}\left(\cap_{n} E_{n}\right)=M C_{m}\left(\cap_{n} E_{n}\right) \cap M C_{1}\left(M C_{m}\left(\cap_{n} E_{n}\right)\right)=$ $M C_{m}\left(\cap_{n} E_{n}\right) \cap M C_{1}\left(\cap_{n} M C_{m}\left(E_{n}\right)\right)=\cap_{n}\left[M C_{m}\left(E_{n}\right) \cap M C_{1}\left(M C_{m}\left(E_{n}\right)\right)\right]=\cap_{n} M C_{m+1}\left(E_{n}\right)$, so it holds for $m+1$ as well. Clearly, (ii) is true whenever both $E$ and $E^{\prime}$ are in $\mathscr{A}$. To prove it in general, take a sequence of events $A_{k}$ such that $E=\cap_{k} A_{k}$ and a sequence of events $A_{k}^{\prime}$ such that $E^{\prime}=\cap_{k} A_{k}^{\prime}$. Then $E=\cap_{k}\left(A_{k} \cap A_{k}^{\prime}\right)$. Since (ii) holds for events, $M C_{1}\left(A_{k} \cap A_{k}^{\prime}\right) \subseteq M C_{1}\left(A_{k}^{\prime}\right)$ for all $k$, hence $M C_{1}(E) \subseteq M C_{1}\left(E^{\prime}\right)$ by part (i). Induction using (ii) shows that $E \subseteq M C_{1}(E)$ implies $E \subseteq M C_{m}(E)$ for every $m$, hence (iii) follows. 
Proof of Proposition 6. Since $\Omega^{\prime}$ is closed, there exist $A_{1}, A_{2}, \ldots$ in $\mathscr{A}$ such that $\Omega^{\prime}=$ $\cap_{n} A_{n}$. From the proof of Proposition 3 we know that $\Omega^{\prime}$ induces a canonical substructure if and only if it satisfies (8). Now (8) implies $\Omega^{\prime} \subseteq M C_{1}\left(A_{n}\right)$ for all $n$, which in turn implies $\Omega^{\prime} \subseteq M C_{1}\left(\Omega^{\prime}\right)$ by part (i) of Lemma 9 , hence $\Omega^{\prime} \subseteq C C\left(\Omega^{\prime}\right)$ by part (iii) of that lemma, hence $\Omega^{\prime}=\Omega^{\prime} \cap C C\left(\Omega^{\prime}\right)$, so that $\Omega^{\prime}$ is a closed component. Conversely, if $\Omega^{\prime}$ is a closed component, then $\Omega^{\prime} \subseteq M C_{1}\left(\Omega^{\prime}\right)$ again by part (i) of Lemma 9 and, by part (ii) of the lemma, $\Omega^{\prime} \subseteq M C_{1}\left(A_{n}\right)$ for all $n$, so $\Omega \backslash A_{n}$ is $\varpi^{i}(\omega)$-null for all $i$, all $n$, and all $\omega \in \Omega$. By Proposition 5 , this implies $\Omega^{\prime}$ satisfies (8).

Proof of Theorem 2. Necessity is an immediate consequence of Proposition 6, whence sufficiency also follows, using Proposition 3 , once we show that every simple and nonredundant structure is minimal. Thus, let $\mathscr{S}=(X, \mathscr{B}, \sigma, \vartheta)$ be a simple and nonredundant structure, let $\gamma$ be its generator, and let $\Omega^{\prime}=\gamma(X)$ be its generated set of hierarchies. Since $(X, \mathscr{B})$ and $(\Omega, \mathscr{A})$ are simple spaces and $\gamma$ is (measurable, hence, by Lemma 3) continuous, and since $\Omega^{\prime}$ is compact and $\mathscr{S}$ is non-redundant, the mapping $\varphi: X \rightarrow \Omega^{\prime}$ such that $x \mapsto \gamma(x)$ is injective, onto, and continuous, hence a homeomorphism (as $X$ is compact and $\Omega^{\prime}$ is Hausdorff). Since $\Omega^{\prime}$ is a compact and hence closed subspace of $\Omega$, by Lemma 1 every subset of $\Omega^{\prime}$ that is both closed and open in $\Omega^{\prime}$ has the form $\Omega^{\prime} \cap A$, where $A \in \mathscr{A}$. Thus, every subset of $X$ that is both closed and open in $X$ (hence every event in $X$, by Lemma 1 ) has the form $\varphi^{-1}\left(\Omega^{\prime} \cap A\right)=\gamma^{-1}(A)$, where $A \in \mathscr{A}$. It follows from Proposition 2 that $\mathscr{S}$ is minimal.

\section{Monotone Continuity and Savage's axioms}

Given a space $(X, \mathscr{B})$, a preference relation $\pi \in \Pi(X, \mathscr{B})$ is monotone continuous if it satisfies the following for all $f, g \in F(X, \mathscr{B})$ and all $z \in Z$.

$M C$ If $(f, g) \dot{\epsilon} \pi$ and $E_{n}$ is a sequence of events in $\mathscr{B}$ such that $E_{n} \downarrow \varnothing$, then $\left(z E_{n} f, g\right) \dot{\epsilon} \pi$ and $\left(f, z E_{n} g\right) \dot{\epsilon} \pi$ for all $n$ sufficiently large.

It is Savage if, for all $f, g, h, h^{\prime} \in F(X, \mathscr{B})$, all $z \in Z$, and all $A, B \in \mathscr{B}$, one has

P1. If $(f, g) \notin \pi$, then $(g, f) \in \pi$. If $(f, g) \in \pi$ and $(g, h) \in \pi$, then $(f, h) \in \pi$.

P2. If $(f A h, g A h) \in \pi$, then $\left(f A h^{\prime}, g A h^{\prime}\right) \in \pi$.

P3. If $A$ is not $\pi$-null, then $\left(z A f, z^{\prime} A f\right) \in \pi$ if and only if $\left(z, z^{\prime}\right) \in \pi$.

P4. If $\left(z, z^{\prime}\right) \dot{\epsilon} \pi$ and $\left(\widetilde{z}, \widetilde{z}^{\prime}\right) \dot{\in} \pi$, then $\left(z A z^{\prime}, z B z^{\prime}\right) \in \pi$ if and only if $\left(\widetilde{z} A \widetilde{z}^{\prime}, \widetilde{z} B \widetilde{z}^{\prime}\right) \in \pi$.

P5. There exist $\bar{z}, \underline{z} \in Z$ such that $(\bar{z}, \underline{z}) \dot{\epsilon} \pi$.

P6. If $(f, g) \dot{\in} \pi$, then there exists a finite partition $\left\{A_{1}, \ldots, A_{N}\right\} \subseteq \mathscr{B}$ of $X$ such that $\left(z A_{n} f, g\right) \dot{\epsilon} \pi$ and $\left(f, z A_{n} g\right) \dot{\epsilon} \pi$ for all $1 \leq n \leq N$.

Note that Savage's seventh axiom, P7 (see Savage 1954), is not needed in our framework, because with finitely many outcomes it is implied by P1 and P3. 


\section{E. Proofs for Section 5}

Proof of Proposition 7. Assume there exists $\pi^{*} \in \Pi_{\text {SAV }}\left(X, \mathscr{B}^{*}\right)$ satisfying (4), let $\left(u, \mu^{*}\right)$ be the SEU representation of $\pi^{*}$, and let $\mu$ denote the restriction of $\mu^{*}$ to $\mathscr{B}$. Since $\mu^{*}$ is finely ranged, so is $\mu$. (For a proof, see e.g. Lemma 6 in Di Tillio 2006) Thus, by (4), the pair $(u, \mu)$ is the SEU representation of $\pi$, hence $\pi \in \Pi_{\text {SAV }}(X, \mathscr{B})$. Conversely, suppose $\pi \in \Pi_{\mathrm{SAV}}(X, \mathscr{B})$ and let $(u, \mu)$ be the SEU representation of $\pi$. By Carathéodory's extension theorem (see e.g. Theorem 3.1 in Billingsley 1995), $\mu$ has a unique extension to a finely ranged belief $\mu^{*}$ on $\mathscr{B}^{*}$. Obviously, the Savage relation $\pi^{*} \in \Pi_{\mathrm{sAV}}\left(X, \mathscr{B}^{*}\right)$ represented by $\left(u, \mu^{*}\right)$ satisfies (4), whereas its uniqueness follows from the uniqueness of SEU representations.

LEMMA 10. $C_{\mathrm{SAV}}^{i}(\Omega) \in \mathscr{A}^{*}$ for every player $i$.

Proof. Fix a player $i$ and write $\Omega_{\mathrm{P} 1}^{i}$ (resp. $\Omega_{\mathrm{P} 2}^{i}, \ldots, \Omega_{\mathrm{P} 6}^{i}$ ) for the set of all $\omega \in \Omega$ such that $\varpi^{i}(\omega)$ satisfies P1 (resp. P2,...,P6). Since monotone continuity is automatically satisfied by every preference relation on $F(\Omega, \mathscr{A})$, we have then $C_{\mathrm{SAV}}^{i}(\Omega)=\Omega_{\mathrm{P} 1}^{i} \cap \cdots \cap \Omega_{\mathrm{P} 6}^{i}$. Define

$$
A_{f, g}^{i}=\left\{\omega \in \Omega:(f, g) \in \varpi^{i}(\omega)\right\}
$$

for all $f, g \in F(\Omega, \mathscr{A})$ and note that, since $F(\Omega, \mathscr{A})$ is countable, there are countably many sets of this form, and by definition of a structure they all belong to $\mathscr{A}$. Thus, it will be enough to prove that $\Omega_{\mathrm{P} 1}^{i}, \ldots, \Omega_{\mathrm{P} 6}^{i}$ can each be obtained from those sets (and their complements) by the usual countable set-theoretic operations. Indeed, the set $\Omega_{\mathrm{P} 1}^{i}$ is the intersection of all sets of the form $A_{f, g}^{i} \cup A_{g, f}^{i}$, where $f, g \in F(\Omega, \mathscr{A})$. The set $\Omega_{\mathrm{P} 2}^{i}$ is the intersection of all sets of the form $A_{f A h, g A h}^{i} \cup\left(\Omega \backslash A_{f A h^{\prime}, g A h^{\prime}}^{i}\right)$, where $f, g, h, h^{\prime} \in F(\Omega, \mathscr{A})$ and $A \in \mathscr{A}$. The set $\Omega_{\mathrm{P} 5}^{i}$ is the union of all sets of the form $A_{z, z^{\prime}}^{i} \cap\left(\Omega \backslash A_{z^{\prime}, z}^{i}\right)$, where $z, z^{\prime} \in Z$. The set $\Omega_{\mathrm{P} 3}^{i}$ is the intersection of all sets of the form

$$
C^{i}(\Omega \backslash A) \cup\left[\left(\left(\Omega \backslash A_{z, z^{\prime}}^{i}\right) \cup A_{z A f, z^{\prime} A f}^{i}\right) \cap\left(A_{z, z^{\prime}}^{i} \cup\left(\Omega \backslash A_{z A f, z^{\prime} A f}^{i}\right)\right)\right],
$$

where $A \in \mathscr{A}, z, z^{\prime} \in Z$, and $f \in F(\Omega, \mathscr{A})$. (Recall that for every $A \in \mathscr{A}$ the set $C^{i}(A)$ is closed, so it is itself a countable intersection of events in $\mathscr{A}$.) The set $\Omega_{\mathrm{P} 4}^{i}$ is the intersection of all sets of the form

$$
\left(\Omega \backslash A_{z, z^{\prime}}^{i}\right) \cup A_{z^{\prime}, z}^{i} \cup\left(\Omega \backslash A_{z^{\prime \prime}, z^{\prime \prime \prime}}^{i}\right) \cup A_{z^{\prime \prime \prime}, z^{\prime \prime}}^{i} \cup\left(\Omega \backslash A_{z A z^{\prime}, z A^{\prime} z^{\prime}}\right) \cup A_{z^{\prime \prime}} A z^{\prime \prime \prime}, z^{\prime \prime} A^{\prime} z^{\prime \prime \prime}
$$

where $z, z^{\prime}, z^{\prime \prime}, z^{\prime \prime \prime} \in Z$ and $A, A^{\prime} \in \mathscr{A}$. Finally, writing $\mathscr{P}_{N}$ for the family of all partitions of $\Omega$ into $N$ events in $\mathscr{A}$, we see that $\Omega_{\mathrm{P} 6}$ is the intersection of all sets of the form

$$
\bigcup_{\left\{A_{1}, \ldots, A_{N}\right\} \in \mathscr{P}_{N}} \bigcap_{1 \leq n \leq N}\left[\left(\Omega \backslash A_{f, g}\right) \cup A_{g, f} \cup\left(C_{z A_{n} f, g} \cap C_{f, z A_{n} g}\right)\right]
$$

where $z \in Z$ and $f, g \in F(\Omega, \mathscr{A})$. (Since $\mathscr{A}$ is countable, so is $\mathscr{P}_{N}$ for every $N$.) 
Lemma 11. For every player $i$ let $\varpi^{* i}: C_{\mathrm{SAV}}^{i}(\Omega) \rightarrow \Pi_{\mathrm{SAV}}\left(\Omega, \mathscr{A}^{*}\right)$ be such that

$$
\varpi^{* i}(\omega)=\varpi^{* i}(\omega) \cap[F(\Omega, \mathscr{A}) \times F(\Omega, \mathscr{A})] \quad \forall \omega \in C_{\mathrm{SAV}}^{i}(\Omega)
$$

and, for every $\omega \in C_{\mathrm{SAV}}^{i}(\Omega)$, let $\left(v^{i}(\omega), \beta^{i}(\omega)\right)$ be the SEU representation of $\varpi^{* i}(\omega)$. Then, for every player $i$, one has

$$
\begin{array}{ll}
\left\{\omega \in \Omega: \omega \in C_{\mathrm{SAV}}^{i}(\Omega), \beta^{i}(\omega)[E]>p\right\} \in \mathscr{A}^{*} & \forall E \in \mathscr{A}^{*}, \forall p \in[0,1] \\
\left\{\omega \in \Omega: \omega \in C_{\mathrm{SAV}}^{i}(\Omega), v^{i}(\omega)[z]>u\right\} \in \mathscr{A}^{*} & \forall z \in Z, \forall u \in \mathbb{R} \\
\left\{\omega \in \Omega: \omega \in C_{\mathrm{SAV}}^{i}(\Omega),(f, g) \in \varpi^{* i}(\omega)\right\} \in \mathscr{A}^{*} & \forall f, g \in F\left(\Omega, \mathscr{A}^{*}\right) .
\end{array}
$$

Proof. Fix a player $i$. Let $\mathscr{A}^{+}$be the family of all $E \in \mathscr{A}^{*}$ satisfying

$$
\left\{\omega \in \Omega: \omega \in C_{\mathrm{SAV}}^{i}(\Omega), \beta^{i}(\omega)[E]>p\right\} \in \mathscr{A}^{*} \quad \forall p \in[0,1] .
$$

We prove the result in three steps. In the first step we prove that $\mathscr{A} \subseteq \mathscr{A}^{+}$. In the second step we prove that $\mathscr{A}^{+}$is closed under the formation of complements and countable monotone unions (hence also countable monotone intersections). By Halmos's monotone class theorem (see e.g. Theorem 3.4 in Billingsley 1995), these two steps together imply $\mathscr{A}^{+}=\mathscr{A}^{*}$ and hence (17). In the third step, we prove that $v^{i}$ satisfies (18). Since (19) clearly follows from (17) and (18), then the proof will be complete.

Step 1. By definition of preference structure,

$$
\left\{\omega \in \Omega:(f, g) \in \varpi^{i}(\omega)\right\} \in \mathscr{A} \quad \forall f, g \in F(\Omega, \mathscr{A}) .
$$

Thus, using (16) and Lemma 10,

$$
\left\{\omega \in \Omega: \omega \in C_{\mathrm{SAV}}^{i}(\Omega),(f, g) \in \lambda^{i}(\omega)\right\} \in \mathscr{A}^{*} \quad \forall f, g \in F(\Omega, \mathscr{A}) .
$$

By Proposition 7 , for all $\omega \in C_{\mathrm{SAV}}^{i}(\Omega)$, the restriction of $\beta^{i}(\omega)$ to $\mathscr{A}$ represents, together with some utility function, the Savage preference relation on $F(\Omega, \mathscr{A})$ defined as

$$
\lambda^{i}(\omega) \cap[F(\Omega, \mathscr{A}) \times F(\Omega, \mathscr{A})] .
$$

For all $E \in \mathscr{A}$ and all $n \geq 1$, write $\mathscr{P}_{E}^{n}$ for the family of all partitions of $E$ into $n$ events in $\mathscr{A}$. Note that, since $\mathscr{A}$ is countable, $\mathscr{P}_{E}^{n}$ is countable. Now fix $E \in \mathscr{A}$ and $p \in \mathbb{R}$. By the proof of Savage's theorem for algebras (Theorem 3.1 in Kopylov 2007),

$$
\beta^{i}(\omega)[E]=\sup \left\{\sum_{A \in P} \frac{1}{\xi^{i}(\omega)[A]}: n \geq 1, P \in \mathscr{P}_{E}^{n}\right\} \quad \forall \omega \in C_{\mathrm{SAV}}^{i}(\Omega),
$$

where for every $A \in \mathscr{A}$ and every $\omega \in C_{\mathrm{SAV}}^{i}(\Omega)$ the integer $\xi^{i}(\omega)[A]$ is defined as follows. Using the fact that the relation (23) satisfies P5, choose any $\bar{z}, \underline{z} \in Z$ such that $(\bar{z}, \underline{z}) \dot{\epsilon}$ $\lambda^{i}(\omega)$. Then $\xi^{i}(\omega)[A]$ is the smallest $m \geq 2$ such that $\left(\bar{z} A \underline{z}, \bar{z} A^{\prime} \underline{z}\right) \dot{\in} \lambda^{i}(\omega)$ for some $P \in$ $\mathscr{P}_{\Omega}^{m}$ and every $A^{\prime} \in P .{ }^{29}$ Thus, the set

$$
\left\{\omega \in \Omega: \omega \in C_{\mathrm{SAV}}^{i}(\Omega), \beta^{i}(\omega)[E]>p\right\}
$$

\footnotetext{
${ }^{29}$ If there is no such integer, let $\xi^{i}(\omega)[A]=+\infty$. In fact, $\xi^{i}(\omega)[A]$ is also the unique (when it exists, that is, when $\left.\beta^{i}(\omega)[A]>0\right)$ integer $m \geq 1$ such that $1 / m<\beta^{i}(\omega)[A] \leq 1 /(m-1)$.
} 
is identical to the set

$$
\bigcup_{n \geq 1} \bigcup_{P \in \mathscr{P}_{E}^{n}}\left\{\omega \in \Omega: \omega \in C_{\mathrm{SAV}}^{i}(\Omega), \sum_{A \in P} \frac{1}{\xi^{i}(\omega)[A]}>p\right\} .
$$

It is therefore clear that, in order to prove $\mathscr{A} \subseteq \mathscr{A}^{+}$, it suffices to show that

$$
\left\{\omega \in \Omega: \omega \in C_{\mathrm{SAV}}^{i}(\Omega), \xi^{i}(\omega)[A] \leq M\right\} \in \mathscr{A}^{*} \quad \forall A \in \mathscr{A}, \forall M \geq 2 .
$$

Thus, fix $A \in \mathscr{A}$ and $M \geq 2$. For all $z, z^{\prime} \in Z$, write $A_{z, z^{\prime}}$ for the set

$$
\left\{\omega \in \Omega: \omega \in C_{\mathrm{SAV}}^{i}(\Omega),\left(z, z^{\prime}\right) \dot{\epsilon} \lambda^{i}(\omega)\right\}
$$

and $B_{z, z^{\prime}}$ for the set

$$
\bigcup_{1 \leq m \leq M} \bigcup_{P \in \mathscr{P}_{\Omega}^{m}} \bigcap_{A^{\prime} \in P}\left\{\omega \in \Omega: \omega \in C_{\mathrm{SAV}}^{i}(\Omega),\left(z A z^{\prime}, z A^{\prime} z^{\prime}\right) \in \lambda^{i}(\omega)\right\} .
$$

By (22), these sets belong to $\mathscr{A}^{*}$. Moreover, by definition of $\xi^{i}(\omega)[A]$,

$$
\left\{\omega \in \Omega: \omega \in C_{\mathrm{SAV}}^{i}(\Omega), \xi(\omega)[A] \leq M\right\}=\bigcup_{z, z^{\prime} \in Z}\left(A_{z, z^{\prime}} \cap B_{z, z^{\prime}}\right)
$$

hence (24) follows.

Step 2. Choose $E \in \mathscr{A}^{+}$and $p \in \mathbb{R}$. Clearly, the set

$$
\left\{\omega \in \Omega: \omega \in C_{\mathrm{SAV}}^{i}(\Omega), \beta^{i}(\omega)[\Omega \backslash E]>p\right\}
$$

is identical to the set

$$
\bigcup_{k \geq 1}\left\{\omega \in \Omega: \omega \in C_{\mathrm{SAV}}^{i}(\Omega), \beta^{i}(\omega)[E] \leq 1-p-1 / k\right\} .
$$

Since $E \in \mathscr{A}^{+}$, each set in the union is the complement of a set of the form (20). Thus, the union is itself in $\mathscr{A}^{*}$, so $\Omega \backslash E \in \mathscr{A}^{+}$and hence $\mathscr{A}^{+}$is closed under the formation of complements. Next, take a sequence $A_{n}$ from $\mathscr{A}^{+}$such that $A_{n} \subseteq A_{n+1}$ for every $n$, and let $A=\cup_{n} A_{n}$. Since $\beta^{i}(\omega)$ is countably additive for every $\omega \in C_{\mathrm{SAV}}^{i}(\Omega)$,

$$
\left\{\omega \in \Omega: \omega \in C_{\mathrm{SAV}}^{i}(\Omega), \beta^{i}(\omega)[A]>p\right\}=\bigcup_{n \geq 1}\left\{\omega \in \Omega: \omega \in C_{\mathrm{SAV}}^{i}(\Omega), \beta^{i}(\omega)\left[A_{n}\right]>p\right\} .
$$

Since $A_{n} \in \mathscr{A}^{+}$for all $n$, the right-hand side is a countable union of elements of $\mathscr{A}^{*}$, hence itself an element of $\mathscr{A}^{*}$. Thus $A \in \mathscr{A}^{+}$and $\mathscr{A}^{+}$is also closed under the formation of countable monotone unions.

Step 3. Pick a bijection $v: Z \times Z \rightarrow\left\{1, \ldots,|Z|^{2}\right\}$. For all $n=1, \ldots,|Z|^{2}$, write $z_{n}$ and $z_{n}^{\prime}$ for the two outcomes satisfying $v^{-1}(n)=\left(z_{n}, z_{n}^{\prime}\right)$ and define

$$
A_{n}^{i}=\left\{\omega \in \Omega: \omega \in C_{\mathrm{SAV}}^{i}(\Omega),\left(z_{n}, z_{n}^{\prime}\right) \in \lambda^{i}(\omega)\right\}
$$


Define recursively $B_{1}^{i}=A_{1}^{i}$ and $B_{n+1}^{i}=A_{n+1}^{i} \backslash\left(B_{1}^{i} \cup \cdots \cup B_{n}^{i}\right)$ for all $n=1, \ldots,|Z|^{2}$. Note that, since $\mathscr{A} \subseteq \mathscr{A}^{*}$, by (16) and (21) we have

$$
B_{n}^{i} \in \mathscr{A}^{*} \quad \forall n=1, \ldots,|Z|^{2} .
$$

Then, since $\lambda^{i}(\omega)$ satisfies Savage's P5 for all $\omega \in C_{\mathrm{SAV}}^{i}(\Omega)$, the sequence of events $B_{n}^{i}$ is a partition of $C_{\mathrm{SAV}}^{i}(\Omega)$. To establish (18), fix $z \in Z$ and $a \in \mathbb{R}$. Clearly, we may assume $0 \leq a<1$. We have

$$
\left\{\omega \in \Omega: \omega \in C_{\mathrm{SAV}}^{i}(\Omega), v^{i}(\omega)[z]>a\right\}=\bigcup_{1 \leq n \leq|Z|^{2}}\left\{\omega \in \Omega: \omega \in B_{n}^{i}, v^{i}(\omega)[z]>a\right\} .
$$

We prove that the set on the right-hand side of the latter belongs to $\mathscr{A}^{*}$, using the fact that for all $\omega \in C_{\mathrm{SAV}}^{i}(\Omega)$ the restriction of $\beta^{i}(\omega)$ to $\mathscr{A}$ is finely ranged. For every $n=$ $1, \ldots,|Z|^{2}$ and every $\omega \in B_{n}^{i}$, the inequality $v^{i}(\omega)[z]>a$ is true if, and only if, ${ }^{30}$ there exists $A \in \mathscr{A}$ such that $v^{i}(\omega)[z]>\beta^{i}(\omega)[A]>a$. The first inequality is equivalent to $\left(z, z_{n} A z_{n}^{\prime}\right) \in \lambda^{i}(\omega)$, so the right-hand side of (26) is

$$
\bigcup_{1 \leq n \leq|Z|^{2}} \bigcup_{A \in \mathscr{A}}\left\{\omega \in \Omega: \omega \in B_{n}^{i}, \beta^{i}(\omega)[A]>a,\left(z, z_{n} A z_{n}^{\prime}\right) \in \lambda^{i}(\omega)\right\} .
$$

Thus, (25), (17), and (22) imply that the right-hand side of (26) belongs to $\mathscr{A}^{*}$.

Proof of Proposition 8. Fix an event $E \in \mathscr{A}^{*}$ and a player $i \in I$. For every $\omega \in C_{\mathrm{SAV}}^{i}(\Omega)$, let $\varpi^{* i}(\omega) \in \Pi_{\mathrm{SAV}}\left(\Omega, \mathscr{A}^{*}\right)$ be the extension of $\varpi^{i}(\omega)$ given by Proposition 7 and let $\beta^{i}(\omega)$ be the belief in the SEU representation of $\varpi^{* i}(\omega)$, so that $\Omega \backslash E$ is null according to $\varpi^{* i}(\omega)$ if and only if $\beta^{i}(\omega)[E]=1$. Then, by the fact that $\beta^{i}(\omega)$ is countably additive for all $\omega \in C_{\mathrm{SAV}}^{i}(\Omega)$, we have

$$
C_{\mathrm{SAV}}^{i}(E)=\bigcap_{k \geq 1}\left\{\omega \in \Omega: \omega \in C_{\mathrm{SAV}}^{i}(\Omega) \text { and } \beta^{i}(\omega)[E]>1-1 / k\right\}
$$

Since each element of this intersection belongs to $\mathscr{A}^{*}$ by Lemma 11 , the result follows.

The proof of Proposition 9 uses the following result.

Lemma 12. Let $E, E^{\prime}, E_{1}, E_{2}, \ldots$ be events in $\mathscr{A}^{*}$. Then

(i) $M C_{\mathrm{SAV}, m}\left(\cap_{n} E_{n}\right)=\cap_{n} M C_{\mathrm{SAV}, m}\left(E_{n}\right)$ for every $m \geq 1$.

(ii) If $E \subseteq E^{\prime}$, then $M C_{\mathrm{SAV}, 1}(E) \subseteq M C_{\mathrm{SAV}, 1}\left(E^{\prime}\right)$.

(iii) If $E \subseteq M C_{\mathrm{SAV}, 1}(E)$, then $E \subseteq C C_{\mathrm{SAV}}(E)$.

Proof. The result follows immediately from the countable additivity of beliefs in SEU representations, using the same arguments as in the proof of Proposition 6.

\footnotetext{
${ }^{30}$ The fact that the restriction of $\beta^{i}(\omega)$ to $\mathscr{A}$ is finely ranged is what guarantees necessity here.
} 
Proof of Proposition 9. Suppose $E$ is a Savage component and pick $D \in \mathscr{A}^{*}$ such that $E=D \cap C C_{\mathrm{SAV}}(D)$. Then $E \subseteq M C_{\mathrm{SAV}, 1}(D) \cap M C_{\mathrm{SAV}, 1}\left(M C_{\mathrm{SAV}, m}(D)\right)$ for all $m \geq 1$, so $E \subseteq M C_{\mathrm{SAV}, 1}(E)$ by part (i) of Lemma 12. Conversely, suppose $E \subseteq M C_{\mathrm{SAV}, 1}(E)$. Then an immediate induction shows that $E \subseteq C C_{\mathrm{SAV}}(E)$ and hence $E \subseteq E \cap C C_{\mathrm{SAV}}(E)$. This proves that (i) and (ii) are equivalent. We now show that (ii) and (iii) are equivalent, thus completing the proof. For every $i \in I$ and every $\omega \in C_{\mathrm{SAV}}^{i}(\Omega)$, let $\varpi^{* i}(\omega)$ be the extension of $\varpi^{i}(\omega)$ to $F\left(\Omega, \mathscr{A}^{*}\right)$ and let $\left(v^{i}(\omega), \beta^{i}(\omega)\right)$ be the SEU representation of $\varpi^{* i}(\omega)$. Then $E$ satisfies (ii) if and only if it satisfies both $E \subseteq M C_{\mathrm{SAV}, 1}(\Omega)$ and

$$
(f E g, f) \in \varpi^{* i}(\omega) \quad \forall i \in I, \forall \omega \in E, \forall f, g \in F\left(\Omega, \mathscr{A}^{*}\right) .
$$

Equivalently, $E$ satisfies (ii) if and only if it satisfies both $E \subseteq M C_{\mathrm{SAV}, 1}(\Omega)$ and

$$
\beta^{i}(\omega)[\Omega \backslash E]=0 \quad \forall i \in I, \forall \omega \in E .
$$

Suppose $E$ satisfies (ii). Then (27) implies, in particular, $(f E g, g) \in \varpi^{i}(\omega)$ for all $i \in$ $I$, all $\omega \in E$, and all $f, g \in F(\Omega, \mathscr{A})$. By Proposition 3 we conclude that $E$ satisfies (iii). Conversely, suppose $E$ satisfies (iii). Then, again by Proposition 3, we must have $\left(z E z^{\prime}, z E z^{\prime \prime}\right) \in \varpi^{i}(\omega)$ for all $i \in I$, all $\omega \in E$, and all $z, z^{\prime}, z^{\prime \prime} \in Z$. But this means that $\beta^{i}(\omega)[\Omega \backslash E]\left(v^{i}(\omega)\left[z^{\prime}\right]-v^{i}(\omega)\left[z^{\prime \prime}\right]\right) \geq 0$ for all $i \in I$, all $\omega \in E$, and all $z^{\prime}, z^{\prime \prime} \in Z$, which-by the fact that $\max _{z \in Z} v^{i}(\omega)[z]=1$ and $\min _{z \in Z} v^{i}(\omega)[z]=0$ for all $i \in I$ and all $\omega \in E$-is possible only if (28) is true, i.e. only if $E$ satisfies (ii).

Proof of Proposition 10. It suffices to prove that $\mathscr{B}$ generates $\mathscr{B}$. Indeed, being the minimal structure associated with a non-redundant structure, $(X, \underline{\mathscr{B}}, \sigma, \underline{\vartheta})$ is obviously non-redundant, whereas from Proposition 7 and from the fact that $\mathscr{B}$ generates $\mathscr{B}$ it immediately follows that it is Savage. Let $\gamma$ be the generator of $(X, \mathscr{B}, \sigma, \vartheta)$. The spaces $(X, \mathscr{B})$ and $\left(\Omega, \mathscr{A}^{*}\right)$ are standard Borel and $\gamma: X \rightarrow \Omega$ is injective (by non-redundancy and by the second part of Proposition 2) and measurable $\mathscr{B} / \mathscr{A} *$ (by measurability $\mathscr{B} / \mathscr{A}$ and by the fact that $\mathscr{B}$ is a $\sigma$-algebra). Since injective functions between standard Borel spaces map events into events, ${ }^{31}$ we have $\gamma(E) \in \mathscr{A}^{*}$ for all $E \in \mathscr{B}$. (Note that, in particular, we have $\gamma(X) \in \mathscr{A}^{*}$.) By injectivity of $\gamma$, this implies that a subset of $X$ belongs to $\mathscr{B}$ if and only if it has the form $\gamma^{-1}(A)$ for some $A \in \mathscr{A}^{*}$. Equivalently, $\mathscr{B}$ is the $\sigma$-algebra generated by the algebra $\left\{\gamma^{-1}(A): A \in \mathscr{A}\right\}$. By the first part of Proposition 2, this family is precisely $\underline{\mathscr{B}}$, so we are done.

Proof of Theorem 3. The second statement, i.e. the uniqueness of $\mathscr{X}$, is an immediate consequence of Propositions 7 and 10 and of the first statement, which we now prove. To establish sufficiency, let $\mathscr{X}=(X, \mathscr{B}, \sigma, \vartheta)$ be a non-redundant standard Savage structure, let $\gamma$ be its generator, and let $\underline{\mathscr{X}}=(X, \underline{\mathscr{B}}, \sigma, \underline{\vartheta})$ be its associated minimal structure. Then $\underline{X}$ is minimal and, by Proposition 10, non-redundant and Savage, hence isomorphic to a Savage canonical substructure. By Proposition 3, this canonical substructure is induced by $\gamma(X)$. Moreover, by the proof of Proposition 10, we have $\gamma(X) \in \mathscr{A}^{*}$. We conclude, by Proposition 9, that $\underline{\mathscr{X}}$ is isomorphic to the canonical substructure induced

\footnotetext{
${ }^{31}$ See, for instance, Corollary 15.2 in Kechris (1995).
} 
by a Savage component. To establish necessity, fix a structure isomorphic to a Savage component; we may of course assume that this structure is in fact a Savage canonical substructure $\left(\Omega, \mathscr{A}^{\prime}, \varrho_{0}^{\prime}, \varpi^{\prime}\right)$ such that $\Omega^{\prime} \in \mathscr{A}^{*}$. Let $\mathscr{A}^{\prime *}$ be the $\sigma$-algebra generated by $\mathscr{A}^{\prime}$. Since $\Omega^{\prime} \in \mathscr{A}^{*}$, and since $\left(\Omega, \mathscr{A}^{*}\right)$ is standard Borel, $\left(\Omega^{\prime}, \mathscr{A}^{*}\right)$ is also standard Borel. Thus, defining $\varpi^{\prime *}: \Omega^{\prime} \rightarrow \Pi_{\mathrm{SAV}}^{I}\left(\Omega^{\prime}, \mathscr{A}^{\prime *}\right)$ from $\varpi^{\prime}$ via Proposition 7 , we obtain the standard Savage structure $\left(\Omega, \mathscr{A}^{*}, \varrho_{0}^{\prime}, \varpi^{* *}\right)$.

Proof of Proposition 11. The first statement is an immediate consequence of Proposition 7 and Lemma 11. The second statement follows at once from the definitions of non-redundancy (for structures and for type spaces) and again from Lemma 11.

\section{REFERENCES}

Aliprantis, Charalambos D. and Kim C. Border (1999), Infinite Dimensional Analysis, second edition. Springer, Berlin. [297]

Arrow, Kenneth J. (1970), Essays in the Theory of Risk-Bearing. North-Holland, Amsterdam. [288, 300]

Aumann, Robert J. (1974), “Subjectivity and correlation in randomized strategies.” Journal of Mathematical Economics, 1, 67-96. [307]

Aumann, Robert J. (1987), "Correlated equilibrium as an expression of Bayesian rationality.” Econometrica, 55, 1-18. [289]

Billingsley, Patrick (1995), Probability and Measure, third edition. Wiley, New York. [317, 318]

Bourbaki, Nicolas (1989), General topology. Chapters 1-4. Elements of Mathematics (Berlin), Springer-Verlag, Berlin. [310]

Brandenburger, Adam and Eddie Dekel (1993), "Hierarchies of beliefs and common knowledge." Journal of Economic Theory, 59, 189-198. [289, 292]

Dekel, Eddie, Drew Fudenberg, and Stephen Morris (2007), "Interim correlated rationalizability." Theoretical Economics, 2, 15-40. [293]

Di Tillio, Alfredo (2006), “Subjective expected utility in games.” Working Paper 311, Innocenzo Gasparini Institute for Economic Research, Università Bocconi. [289, 292, 305, 306, 317]

Ely, Jeffrey C. and Marcin Pęski (2006), "Hierarchies of belief and interim rationalizability.” Theoretical Economics, 1, 19-65. [293]

Epstein, Larry G. and Tan Wang (1996), “'Beliefs about beliefs' without probabilities.” Econometrica, 64, 1343-1373. [288, 289, 292, 303, 304]

Forges, Françoise (1993), "Five legitimate definitions of correlated equilibrium in games with incomplete information.” Theory and Decision, 35, 277-310. [307] 
Gul, Faruk (1992), “Savage's theorem with a finite number of states." Journal of Economic Theory, 57, 99-110. See also "Erratum”, Journal of Economic Theory, 61, 184. [300]

Harsanyi, John C. (1967-68), “Games with incomplete information played by ‘Bayesian’ players, Parts I, II, III.” Management Science, 14, 159-182, 320-334, and 486-502. [288]

Heifetz, Aviad and Dov Samet (1998), “Topology-free typology of beliefs.” Journal of Economic Theory, 82, 324-341. [295]

Kechris, Alexander S. (1995), Classical Descriptive Set Theory. Springer-Verlag, New York. [302, 303, 321]

Koppelberg, Sabine (1989), Handbook of Boolean Algebras, volume 1. North-Holland, Amsterdam. [308]

Kopylov, Igor (2007), “Subjective probabilities on 'small' domains.” Journal of Economic Theory, 133, 236-265. [300, 318]

Lindenstrauss, Joram (1966), “A short proof of Liapounoff's convexity theorem.” Journal of Mathematics and Mechanics, 15, 971-972. [300]

Mertens, Jean-François and Shmuel Zamir (1985), "Formulation of Bayesian analysis for games with incomplete information." International Journal of Game Theory, 14, 1-29. [288, 289, 292, 293, 295, 303, 307]

Savage, Leonard J. (1954), The Foundations of Statistics. Wiley, New York. [287, 300, 316]

Villegas, C. (1964), “On qualitative probability $\sigma$-algebras.” Annals of Mathematical Statistics, 35, 1787-1796. [288, 300]

Submitted 2006-11-2. Final version accepted 2008-5-22. Available online 2008-5-22. 